\title{
The Star Formation History of the Carina Dwarf Galaxy
}

\author{
Denise Hurley-Keller \& Mateo Mateo \\ Astronomy Department, University of Michigan \\ James Nemec \\ International Statistics \& Research Corporation
}

\begin{abstract}
We have analyzed deep $B$ and $V$ photometry of the Carina dwarf spheroidal reaching below the old main-sequence turnoff to $V \sim 25$. Using simulated color-magnitude diagrams to model a range of star formation scenarios, we have extracted a detailed, global star formation history. Carina experienced three significant episodes of star formation at $\sim 15$ Gyr, 7 Gyr, and 3 Gyr. Contrary to the generic picture of galaxy evolution, however, the bulk of star formation, at least 50\%, occured during the episode 7 Gyr ago, which may have lasted as long as 2 Gyr. For unknown reasons, Carina formed only 10-20\% of its stars at an ancient epoch and then remained quiescent for more than 4 Gyr. The remainder $(\sim 30 \%)$ formed relatively recently, only 3 Gyr ago. Interest in the local population of dwarf galaxies has increased lately due to their potential importance in the understanding of faint galaxy counts. We surmise that objects like Carina, which exhibits the most extreme episodic behavior of any of the dwarf spheroidal companions to the Galaxy, are capable of contributing to the observed excess of blue galaxies at $B \sim 24$ only if the star formation occurred instantaneously.
\end{abstract}

Subject headings: galaxies: evolution, galaxies: stellar content

\section{Introduction}

The Carina dwarf galaxy, one of the nine known dwarf spheroidal companions to the Galaxy, has a surprisingly complex star formation history. Detection of carbon stars provided the first suggestion of a significant intermediate-age population (Cannon, Niss, \& Norgard-Nielsen 1981), followed by main-sequence photometry which revealed a young turn-off due to a population perhaps only 6-9 Gyr old (Mould \& Aaronson 1983; 
MA hereafter). MA fit a simulated luminosity function comprised of 7 Gyr-old stars to their data, and estimated the contribution of an old population to be relatively small in comparison. Saha, Monet, and Seitzer (1986) observed a large number of RR Lyraes in the central region of the galaxy and established a lower limit of $2-3 \%$ for the fraction of old stars, depending on the yield of RR Lyraes. Mighell (1990) estimated the relative sizes of the populations from the double-peaked color distribution near the MSTO region to be $85 \%$ for the intermediate-age burst and $15 \%$ for the old episode. Using simulated luminosity functions, Mighell \& Butcher (1992) later fit intermediate-age burst models to this deep, main-sequence photometry and estimated an upper limit to the old population of 40\%. More recently, Smecker-Hane, et al. (1994; hereafter SHSHL) resolved two separate horizontal branches (HBs). Although the separation is not independent of metallicity effects, it is very suggestive of the multi-episode nature of Carina's history.

We are left with an estimate between $2 \%$ and $40 \%$ for the fraction of old stars, and no clear result on the duration of the star formation episodes. The chemical evolution of the galaxy is likewise still in question. Spectroscopy of 15 giants in Carina (Da Costa 1994) resulted in an average $[\mathrm{Fe} / \mathrm{H}]$ of -1.9 , with one giant significantly more metal poor $([\mathrm{Fe} / \mathrm{H}]$ $=-2.2$. The excellent areal coverage of the SHSHL data reveal a very thin giant branch. Given the huge apparent age spread, a metallicity spread may be necessary to compensate and produce the observed narrow RGB.

Improved methods of analysis are needed. Detailed analyses of stellar luminosity functions have some advantages over more traditional isochrone fitting. These methods, however, were designed for coeval systems. Because dwarfs exhibit a range of ages and metallicities, these approaches are problematic at best, and misleading at worst. A much better way to unravel complex star formation histories like those exhibited by dwarf galaxies such as Carina and Leo I is to use all of the information embodied by color-magnitude diagrams. In these cases, where the galaxies appear to have experienced bursts at intermediate epochs, a conventional luminosity function would not distinguish between the old subgiant branch and the young MS stars, for example. Color information proves essential to resolve multiple components in these systems.

Simulated color-magnitude diagrams have been used successfully in studies of bright stars in dwarf irregulars (Tosi et al. 1991; Tolstoy 1996; Gallart et al. 1996a, 1996b, Aparicio et al. 1997a, 1997b), of LMC field stars (Bertelli et al. 1992; Vallenari et al. 1996), and of LMC clusters (Vallenari et al. 1994). We have deep CCD photometry (limiting magnitude $V=24.5)$ with reasonably small photometric errors $(\sim 0.02$ at $V=23)$ of three fields in the Carina dwarf galaxy, reaching well below the old main-sequence turnoff. Because the photometry reaches below the old MSTO, these data are well-suited to a 
detailed extraction of the star formation history by comparing model color-magnitude diagrams to that of Carina.

Section 2 is a discussion of the observations and reductions of the Carina data. Section 3 outlines the analysis applied to these data, including the development of the pseudo-LF and the generation of similulated color-magnitude diagrams. The results, presented in section 4, are summarized and briefly discussed in the context of galaxy evolution in section 5.

\section{Observations}

\subsection{Photometry}

The Carina observations were acquired using a $800 \times 800 \mathrm{TI}$ CCD at the CTIO $4 \mathrm{~m}$ telescope during two runs in March of 1989 and 1990. The observations cover three fields in $B$ and $V$, located as shown in Figure 1 and labelled F1, F2, and F3. Also outlined in this figure is the region (M) observed by Mighell(1990). The pixel scale for this instrument was $0.292^{\prime \prime}$ per pixel, so that each of our fields is $15.16 \mathrm{arcmin}^{2}$. The mediocre seeing permitted us to bin the images $2 \times 2$, so that the final scale was $0.58^{\prime \prime}$ per pixel.

Field 1 was observed in 1989; fields 2 and 3 were observed in 1990. The typical exposure time for each image was $500 \mathrm{~s}$, with the total exposure time in each filter for each field as listed in Table 1. Table 1 also lists the coordinates of the fields and the number of images in each filter. Control field observations, taken $1^{\circ}$ south of Carina, are also summarized in Table 1, and were used to correct the data for contamination due to foreground galactic stars and background galaxies.

The images were processed and reduced using standard techniques. After bias subtraction and flatfielding, the images were registered by calculating frame-to-frame offsets in $\mathrm{x}$ and $\mathrm{y}$ position using bright unsaturated stars. We then coadded all the frames in each filter to obtain deep combined images in both $B$ and $V$ for each field. These combined images were then reduced using DoPHOT (Schechter et al. 1993). Athough the conditions were photometric for both runs, the seeing was worse than $1.2^{\prime \prime}$ for some of the exposures

which undoubtedly affected the quality of the combined images. Also, the first 100 columns of the images were unusable because of a defective anti-reflective coating on the chip; thus we excluded these columns from the reduction. The number of stars detected in each field is shown in Table 1.

Each field was calibrated via observations of Graham standards (Graham 1982) 
which covered an appropriate range in color and airmass. These primary standards were used to calibrate the individual $\mathrm{B}$ and $\mathrm{V}$ frames for each night. In general, the fits to the standard star magnitudes and colors exhibit an rms scatter of $\sigma \leq 0.03$ mag (see Mateo et al. 1991 for a discussion of the calibration of the 1990 data). Secondary standards from each field were then used to calibrate the coadded frames. Table 2 gives a sample of the $B$ and $V$ photometry of the Carina stars. The complete version of Table 2, showing photometry for all the stars detected in all three fields, can be accessed at ftp:/ra.astro.lsa.umich.edu/pub/get/denise/carina.phot. Table 3 lists the internal errors as a function of magnitude as returned by DoPHOT for each field. Figures 2 and 3 show the distributions of error vs. magnitude for each of the three fields in Carina. The individual frames were also reduced and were searched for variable stars; those results are discussed in Mateo et al. (1998).

\subsection{Completeness}

Carina is a completely uncrowded field to the limit of our photometry. The resolved stellar density in the deepest field (field 1) is about 1 resolved star per 52 pixels. The typical stellar image in that field covers an area of 28 pixels. While this does not mean that blending of stellar images or mismatches of stars between filters does not occur at all, it does mean that they happen rarely, and thus likely produce a small effect only on the photometry near the detection limit (taken to be $3 \sigma$ ). In our analysis, we only consider photometry roughly one magnitude or more brighter than the detection limit in our data.

Due to the lack of crowding in the Carina fields, we felt that the observational effects relevant to the determination of the star formation history of Carina could be realistically treated without resorting to the statistical use of artificial stars as described in Aparicio \& Gallart 1995. This highly successful but computationally expensive technique has been applied to several dwarf irregulars in the Local Group, where the stellar density is much higher and the contribution of unresolved light much greater. In our analysis, we will incoporate completeness effects and photometric errors and neglect effects due to crowding.

To this end, we conducted a false-star analysis to determine the completeness as a function of $B-V$ color and $V$ magnitude. For each field, $100 B$ and $V$ image pairs were generated to which 100 false stars had been added. Thus, the number of false stars added to a single frame was less than $10 \%$ of the number of real stars detected, but a total of 30,000 stars were added to all three fields. The final result is based on rejecting stars added to severely saturated regions of the images, as well as to the 100 bad columns. In this way,

we established a statistically sound completeness estimate without significantly altering the 
degree of crowding.

$V$ magnitudes and $B-V$ colors were assigned to the false stars beforehand, and the $B$ and $V$ images were generated simultaneously. Adding false stars to the $B$ and $V$ images separately leads to problems with cross terms when calculating the completeness factor (see Aparicio et al. 1995). The slope of the luminosity function of artificial stars was fixed to be $\sim 0.4$, roughly that of the data, in order to correctly reproduce the effects of bin migration (Mateo 1988). The assigned colors sampled a uniform distribution across the range covered by the data. Positions were also randomly selected from a uniform distribution across the CCD field. DoPHOT was then applied to the these frames in the same manner as to the original data. Figure 4 shows the differences in input and output magnitude plotted against the input magnitude for field 1 . This distribution is not symmetric because, while only random error makes a measured magnitude fainter than an input magnitude, artificial stars can be added on top of an existing faint, real star, causing measured magnitudes to be brighter. While this is a real effect that will cause the errors to deviate from a gaussian distribution, note that there are relatively few (less than 10\%) of these stars with $m_{i}-m_{o}$ much greater than zero at magnitudes brighter than $V \sim 24$.

Finally, the completeness is calculated in a simple rectangular grid in $V$ vs. $(B-V)$ by comparing the number of stars added to a grid element to the number from that grid element which are detected. Eventually, this completeness factor is used by the program which generates synthetic color-magnitude diagrams to decide whether a given star is detected or not. As we will discuss in section 2.4, we found the three fields to have no significant differences, and combined results from them for a quantitative analysis of the global star formation history. The combined completeness for the three fields can be calculated from the results for the individual fields:

$$
C(V, B-V)=\frac{C_{1}+C_{2}+C_{3}}{N_{1}+N_{2}+N_{3}}
$$

where $C$ and $N$ are the completeness and the number of stars added in a given $V,(B-V)$ bin for each of the three fields, respectively. Results of this completeness analysis are shown in Figure 5. We shall incorporate these corrections into the model calculations as described in a later section.

No false star analysis was run on the control field. The completeness was assumed to be the same as for the combined Carina images. This assumption is inaccurate at faint magnitudes, as the overall depth of the control field images is less than that of the Carina images. However, the effect of such an error in the completeness for this field is diminished by the greater density of Carina stars in the region of the CMD used in our analysis, and by the fact that only stars with $V<24.2$ are considered. Any error is likely to have only 
an insignificant effect on the results of our analysis.

\subsection{Comparison with Previous Photometry}

Our photometry shows good agreement with previous photometric studies of Carina. The overall calibration of the color-magnitude diagram for all three fields (Figure 6d) is consistent with the results of MA and Mighell (1990). Both put the location of the red clump at $V \sim 20.5$. The average $V$ magnitude of stars in the clump in the CMD in Figure $6 \mathrm{~d}$ is 20.46. Because of the greater areal coverage of their study, SHSHL were able to identify the blue $\mathrm{HB}$ at $V=20.65 \pm 0.05$. Isolating the sparsely populated extended blue HB in our diagram, the average $V$ is 20.71, which is consistent with the SHSHL result.

As can be seen in Figure 1, our fields 1 and 2 overlap with the field observed by Mighell, and a direct comparison of the photometric datasets can be made. We show the results of matching stars from Mighell observations with those of field 2 (which has the largest overlapping area) in Figure 7. Our photometry is systematically brighter in V by $0.034 \pm 0.003 \mathrm{mag}$. There were two stars with differences $>5$ magnitude; both were faint stars mismatched with nearby bright stars. Most of the other objects with differences $>1$ magnitude either have relatively nearby companions or are obviously mismatched. Although removal of these objects does not remove the systematic difference in the photometry, an error of this size in our photometry, if real, will not affect the final results of our analysis.

We constructed a luminosity function from our data, as well as from those of Mighell (1990) and MA. No correction was made for completeness or foreground/background contamination in any of the datasets for this comparison. The luminosity functions were normalized to the number of stars in the $V$ magnitude range 21.2 to 22.5. Figure 8 shows

reasonable agreement between all three studies until the faintest bins where incompleteness becomes important.

\subsection{Color-Magnitude Diagrams}

Figure 6 shows the color-magnitude diagram for the individual Carina fields and for all three combined (7309 stars). Three MSTOs can be identified in the combined CMD: a "young" MSTO at $V \sim 22.3$, an intermediate-age MSTO at $V \sim 22.7$, and an old MSTO at $V \sim 23.2$. Given the age spread, the narrowness of the RGB at $B-V \sim 0.7$ is suggestive of a metallicity spread as recognized by SHSHL. In particular, note the prominent red clump at $V \sim 20.5$ and the extended blue HB first noted by SHSHL, but only weakly visible in 
this diagram due to the smaller sample of stars. Also visible in the diagram are foreground galactic stars with $B-V>0.6$ and background galaxies with $V>23$ and extending to very blue colors.

Does Carina have a blue straggler population? Many stars in Carina's CMD obey the strict definition of blue stragglers: stars located along the main sequence above the old turn-off in an ancient population. However, we argue that most of these are BS pretenders and that it is much more likely that Carina has formed stars in the recent past $(\sim 1-3$ Gyr). In globular clusters, Preston et al. 1994) found that the ratio of blue stragglers to BHB stars is $\sim 0.6$. Moreover, the luminosity function of blue stragglers in globular clusters increases from a luminosity cutoff at $M_{V} \sim 1.9$ towards the ancient main-sequence turn-off point at $M_{V} \sim 4$ (Sarajedini \& Da Costa (1991); Fusi Pecci et al. 1992); for Carina, this cutoff corresponds to $V \sim 22$. The blue horizontal branch (BHB) is comprised of those stars blueward of the instability strip, the blue edge of which must be near $B-V \sim 0.21$ given the expected reddening towards Carina. In the Carina CMD, there are 10 stars in this region. If blue stragglers of the kind found in globular clusters exist in Carina, there should only be roughly six such stars, and they would be buried among the many stars located along the main sequence between $V \sim 24-22$. An old BS population cannot explain the presence of the pretenders, which number close to 50 and reach to $V \sim 21.2$; only stars younger than 10 Gyr could populate this region of the CMD at such high density.

Several factors led us to conclude that any variation in star formation history between the three fields which we have studied is insignificant. First, the three fields lack an obvious qualitative difference. Visual inspection shows that the same large-scale features visible in the combined CMD, such as the intermediate-age turn-off and the red clump, remain in the CMDs for the individual fields. Second, Figure 9 shows that the luminosity functions for the three fields are similar. Third, the numbers of stars in large regions of the CMD which represent different evolutionary stages are consistent from field to field. Table 4 lists the numbers of stars in the "BS" region, the BHB, the red clump, and in a region at the upper MSTO for each field in Carina. For each region, the numbers are within roughly $2 \sigma$ of the average value for that region. This should be contrasted with recent evidence for real spatial variations in the horizontal branch in some dSph (Da Costa et al. 1996). Ultimately, any differences between the fields are lost due to the small number statistics; there simply are not enough stars in any one field to do the quantitative analysis that is carried out on the combined CMD (see below). 


\section{Analysis}

Interpreting the Carina data involved three basic steps. First, we determined the pseudo-luminosity function (pseudo-LF; defined below) for these data, hoping to avoid the problems of a simple luminosity function analysis. We then generated a set of synthetic color-magnitude diagrams, including photometric errors and completeness effects, which sampled a parameter space appropriate for our data. We determined the pseudo-LF for the model CMDs. Finally, we calculated the $\chi^{2}$ value for the model and data pseudo-LFs and applied a criteria for selecting the star formation scenarios which were most likely to lead to the observed Carina CMD. This method provides increased detail in the derivation of the SFH of Carina at the expense of decreased reliablility in the specific results due to the greater statistical demands placed on the data by this procedure.

\subsection{Determining the Pseudo-LF for Carina}

The analysis of stellar populations in nearby, resolved dwarf spheroidals has been limited in the past to methods applied to globular clusters, which dSph were expected to resemble. Historically, the standard technique has been to combine isochrone fitting (i.e. Flannery \& Johnson 1982) and an analysis of the features of the color-magnitude diagram. Relationships between the observed properties of the color-magnitude diagram (RGB color at the level of the HB, for example) and the underlying evolutionary parameters (metallicity and age, for example) are derived from galactic globular cluster observations, and observations of globular cluster systems in nearby galaxies (Sandage 1982; Lee et al. 1990; Da Costa \& Armandroff 1990, for example). This approach is designed for coeval systems, and is problematic for dwarfs which exhibit an internal range of ages and metallicities.

Detailed analysis of stellar luminosity functions is an improvement at some level. Included in the fitting is the relative numbers of stars at different stages along the isochrone, as well as the shape of the isochrone. In addition, uncertainties in the details of stellar atmosphere models which can affect the colors do not affect the luminosities strongly (Paczynski 1984; Ratcliff 1987). Evolutionary parameters can be derived from the features of the stellar LF. With the availability of moderately fast workstations, grids of simulated LFs for populations with different star formation histories and chemical evolution can be computed for statistical comparison to the observations (Mighell \& Butcher 1992; references therein). This method is appropriate for populations such as globular clusters which experience a single episode of star formation, but luminosity functions for systems with more complex star formation histories are difficult to interpret. Intermediate-age 
populations have been observed in both the Carina and Leo I dwarf spheroidal companions to the Milky Way. In these cases, where the galaxies appear to have experienced bursts at intermediate epochs, a conventional luminosity function would not distinguish between the old subgiant branch and somewhat younger MS stars. Color information proves essential to resolve multiple components in these systems.

We seek to solve this problem by isolating in color the main sequence turn-off region and the subgiant branch, where evolution slows as stars first ascend the RGB. A high degree of resolution in age and metallicity will be achieved in these two areas where the shape of the isochrones are sensitive to age and metallicity and the evolution is relatively slow enough to ensure a statistically significant number of stars in the studied region. In contrast, the structure of the unevolved main sequence is dominated by the mass function, and the RGB by dispersion in color due to the photometry. In the Hertzsprung gap, the magnitude of stars is very sensitive to age and metallicity, but the evolution is rapid, so that in our data there are too few stars in this region for a quantitative analysis.

In a further effort to optimize the sensitivity to dispersion in age and metallicity, a pseudo-luminosity function is constructed in these regions. This function differs from the standard luminosity function in two ways. First, if the bins are all of fixed size, the statistical fluctuations are larger in bins where there are fewer stars. Instead, we use bins of variable size which contain a fixed number of Carina stars. The size of each bin varies and reflects the stellar surface density in the CMD. The number of Carina stars is determined by correcting for the number of contaminants in the bin using the control field photometry; as the number of control stars in a given bin varies, so does the size of the bin. This somewhat complex, iterative procedure ensures a statistically significant number of stars in each bin. Second, the slope of the bins is roughly aligned with the slope of the isochrones in the targeted regions to prevent the blending of stars from regions of different evolutionary stages.

We define the pseudo-luminosity function as the ratio of the number in the bin to the area of the bin, and normalize this to a region which combines the two brightest bins of the subgiant branch pseudo-LF:

$$
R_{i}=\frac{\left(N_{i} / A_{i}\right)}{\left(N_{R G B} / A_{R G B}\right)}
$$

where $A_{i}$ is the area in the indicated bin and $N_{i}$ is the number of stars in that bin. After some experimentation, 50 stars/bin in the MSTO region and 30 stars/bin in the subgiant branch region provided sufficient stars per bin to reduce the statistical fluctuations without smoothing out the important features. The Carina CMD with the bins generated in this manner is shown in Figure 10. 
The pseudo-LFs for the data, in Figure 11, can be interpreted in terms of Carina's $\mathrm{SFH}$. The intermediate-age main sequence turn-off appears as a sudden drop in the MS pseudo-LF at $V \sim 23$. The old turn-off is dominated by the intermediate-age main sequence and completeness, which drops sharply $(\sim 20 \%)$ between $V=23$ and $V=24$. Keeping this in mind, the old turn-off can be discerned at $V \sim 23.5$ where a less distinct drop occurs. The subgiant branch is easier to interpret. The two obvious peaks correspond to an enhancement in the number of stars at roughly 3 Gyr and 7 Gyr. These populations can also be recognized directly in the varying size of the bins along the subgiant branch in Figure 10. The third, sixth, and seventh bins are noticably smaller than the adjacent bins, corresponding to the region where $3 \mathrm{Gyr}$ and $7 \mathrm{Gyr}$ isochrones would join the RGB. The sharp rise at faint magnitudes is where the main sequence begins to affect the bins.

Since a significant population as young as 3 Gyr is not immediately obvious in the Carina CMD, we initially considered the possibility that the brighter peak in the subgiant branch pseudo-LF was the "Thompson bump". A slow-down in the giant branch evolution occurs when the H-burning shell reaches the discontinuity in chemical composition left behind by the recession of the convective envelope (Bergbusch \& VandenBerg (1992) and references therein). This causes a peak in the RGB luminosity function, the position and amplitude of which depend on metallicity. Given that Carina is extremely metal-poor $([\mathrm{Fe} / \mathrm{H}] \sim-2.0$ to $-2.2 \mathrm{dex})$, any "bump" due to the old population should be of small amplitude and should be located roughly two magnitudes brighter than the bright peak in the subgiant branch pseudo-LF (Fusi Pecci et al. 1990). Younger populations produce an even brighter "bump". Thus, neither the old nor the intermediate-age population can provide an explanation for this feature.

\section{2. $\quad$ Generating Simulated CMDs}

The Carina CMD simulations use a set of metal-poor isochrones with a helium fraction $\mathrm{Y}=0.235$, ranging in age from $2-18 \mathrm{Gyr}$ and in metallicity from $[\mathrm{Fe} / \mathrm{H}]=-1.45$ to -2.2 dex. The isochrones, kindly provided to us by Mike Trippico, were generated using a code similar in physics and techniques to that of VandenBerg (1983). While standard techniques

are used to convert evolutionary tracks into isochrones in the fundamental $\log T_{\text {eff }}-\log$ $L$ plane, the colors are determined from detailed synthetic spectra which are computed at intervals along the isochrones (Tripicco, Dorman and Bell, 1992; hereafter TDB). The evolution extends through the first-ascent red giant branch (RGB) and terminates with the He-flash. We do not use the He-burning tracks also discussed in TDB. TDB test the models on M67, a well-studied, old open cluster, and find reasonably good agreement between the 
observed RGB and the theoretical RGB which uses the semi-empirical surface pressure boundary conditions devised by VandenBerg (1992).

Our program generates synthetic CMDs in the following manner. A mass is chosen according to the Salpeter (1955) mass function.

$$
d N=A M^{-x} d M
$$

where $A$ is a normalization factor which depends upon the upper and lower mass limits used in the models. The limits $\left(0.7 M_{\odot}\right.$ to $\left.1.5 M_{\odot}\right)$ bracket the range of masses present in the main sequence and red giant branch $(V \leq 25)$. The slope of the mass function, $x$, is 2.5. We fixed this parameter in order to suppress the number of models generated and because no strong evidence exists for varying the slope in old or intermediate-age stellar populations (Hunter et al. 1997). Different values of the IMF slope would affect most strongly the relative strengths of the populations. The models are normalized to the data using the number of stars in the RGB brighter than $V=22$. This required isolating the red giant branch from the horizontal branch and obvious contamination. Combining the data from all three of the Carina fields yielded 163 stars brighter than $V=22$.

We use a Monte Carlo method to randomly select an age according to either a constant or episodic star formation history. The metallicity follows from a linear age-metallicity relationship established by input parameters. Given our photometric errors and especially the small number of giants in our sample, we cannot easily distinguish between a scenario with no metallicity spread and one with a 0.2 dex enrichment between the old and intermediate-age populations. SHSHL suggested this spread based on the narrowness of their much more densely populated RGB. We chose an $[\mathrm{Fe} / \mathrm{H}]=-2.23$ for the old population and $[\mathrm{Fe} / \mathrm{H}]=-2.0$ for the intermediate-age and young populations. Using a constant $[\mathrm{Fe} / \mathrm{H}]$ for the duration of star formation in Carina is unlikely to change the results of our analysis.

Given a mass, age, and metallicity, the photometric properties of the star are interpolated from the bounding isochrones using equivalent evolutionary points in a method similar to that described in Bergbusch \& VandenBerg (1992). Figure 12 shows an isochrone interpolated from bounding isochrones and one derived from the $\log L-\log T_{\text {eff }}$ plane. At the points of least agreement, the color error is $\leq .02$ and the error in $V$ is $\leq 0.02$.

The program also incorporates observational effects into the CMD. We supply the appropriate magnitude and color errors as a function of magnitude, estimated from the internal errors returned by DoPHOT (see Table 3). The program then randomly assigns error in both magnitude and color independently, assuming a Gaussian distribution. The completeness as a function of both magnitude and color is determined from the false star analysis. A grid of completeness values is supplied to the program. The star is either kept 
or discarded based on the completeness interplolated from the grid given its color and magnitude. Rather than correcting the model color-magnitude diagram for contamination due to background galaxies and foreground stars, we correct the pseudo-luminosity function directly later in the analysis.

The full star formation history of a galaxy is a potentially complex function of time and location. To make the problem manageable, we were forced to limit the range of parameter space which our models covered based on previous results and the suggestions of our own data. The color-magnitude diagram of Carina suggests episodes of strong star formation, bracketed by quiescent periods. Main-sequence turn-offs reveal convincingly the presence of old ( $\sim 15 \mathrm{Gyr})$ and intermediate-age ( $\sim$ Gyr) populations. The inability of any two-episode models to reproduce the brighter peak in Carina's subgiant branch pseudo-LF led us to the suspect the existence of a "young" ( $~ 3$ Gyr) population. As a result, we concluded that three episodes of star formation were responsible for the bulk of Carina's stars. Figure 13 illustrates the parameterization of Carina's star formation history. We focused on extracting the age and SFR information: $t_{i}$ designates the age center of the SF episode, $\Delta_{i}$ the duration of the episode, and $S_{i}$ the relative strength of the episode.

Photometric errors and age resolution conspire to limit our understanding of the old population. The suspected strength of this population $(\leq 30 \%)$ means there would be a relatively small number of old stars which, depending on the episode duration, could be lost in the statistical noise of the main sequence at that magnitude. In addition, the photometric errors cause a 0.5 Gyr-wide episode to appear 2 Gyr-wide in in the CMD. Thus, constraining the episode center and duration is difficult for this population. The pseudo-LF will be sensitive to the population strength, however, as it is normalized to a region on the RGB which will contain old stars. Thus $t_{3}$ and $\Delta_{3}$ are fixed at 15 Gyr and 1 Gyr, respectively, and $S_{3}$ is allowed to vary between $10 \%, 20 \%$, and $30 \%$. While we can still estimate then the contribution of this population to Carina, we cannot precisely determine the implied star formation rate because we can only place an upper limit on the duration of the episode.

For the population near $3 \mathrm{Gyr}$, the fine age resolution helps to limit the parameter space that needs to be covered. Figure 14 shows the subgiant branch pseudo-LF for Carina and three models which have strong 3 Gyr populations. Shifting the center of the episode by 1 Gyr creates a clear effect in the subgiant branch pseudo-LF. As a result, ages of 4 or 2 Gyr are less likely to produce a good fit to the data. 3 Gyr is a reasonable choice for $t_{1}$. The episode duration, $\Delta_{1}$, and strength, $S_{1}$, remain free parameters.

We expect to extract the most information about the intermediate-age $(\sim 7$ Gyr $)$ episode. Previous research as well as the CMD itself indicate that this is the largest 
population in Carina, so that there will be a significant number of stars to populate the bins. In addition, a reasonable degree of age resolution is still possible at this magnitude given the photometric errors. Therefore, $t_{2}, \Delta_{2}$, and $S_{2}$ are free parameters, covering values

appropriate given the appearance of the color-magnitude diagram. Tables 5 and 6 show the range of parameters covered by the 180 models generated for this analysis.

Photometry of a large number of stars is needed to damp the statistical poisson noise. Figure 15a shows the pseudo-LFs of five models with the same star formation history with roughly the same number of stars as the Carina data. The large amount of variation between these models is apparent. The statistical variations between them limit how well we can distinguish between distinct models. We cannot easily determine what effects are due to statistical variation and what to the difference in star formation history. Figure 15b shows the pseudo-LFs for five models with the same star formation history, but each with ten times as many stars as the Carina data. As expected, the variations are much smaller. Thus, using a model ten times the size of our data set, we are reducing the relative size of the errors on the model pseudo-LFs, and improving the sensitivity of our method. Each of the model CMDs in Table 6 has 1630 stars in the RGB above $V=22$.

Finally, the model RGBs appeared about 0.04 magnitudes bluer than the data. The exact cause of the shift is unknown, although several possibilities exist. The Trippico et al. isochrones are derived from Vandenberg isochrones, which are historically believed to be too blue by roughly the observed amount (Vandenberg 1983). Another possible source of discrepancy is the calibration of our photometry, which was found to be systematically brighter by about 0.03 magnitudes than Mighell's $V$ photometry. If Mighell's magnitudes are correct, then assuming our $B$ magnitudes are correct, our $B-V$ colors would be too red by 0.03. Lastly, our chosen values for $z$ in the synthetic CMDs could be too metal poor to accurately describe the data. Whatever the source of the discrepancy, the bins as applied to the models are shifted in color 0.04 magnitudes to the blue. Without the shift, fits for all the models are uniformly worse. Within the framework of our approach, one could consider the color shift to be an additional global parameter that we have to determine.

\subsection{Comparing the Data and the Models}

The $\chi^{2}$ statistic can be used to compare the pseudo-LFs of the models and the data.

$$
\chi^{2}=\frac{\left(R_{\text {data }}-R_{\text {model }}\right)^{2}}{\sigma_{\text {data }}^{2}+\sigma_{\text {model }}^{2}}
$$

where $R_{\text {data }}$ is the ratio measured from the data, $R_{\text {model }}$ is that measured from the model, $\sigma_{\text {data }}$ is the error in $R_{\text {data }}$, and $\sigma_{\text {model }}$ is the error in $R_{\text {data }}$. Both $\sigma_{\text {data }}$ and $\sigma_{\text {model }}$ are 
calculated assuming Poisson counting errors for the pseudo-LF bins.

We tested the ability of our method to correctly identify the underlying star formation scenario by generating ten models with the same input SFH parameters and roughly the same number of RGB stars as the Carina data. Each of these ten sets of data were treated as real data; i.e. bins were determined so that the same number of stars were in each bin, and these bins were then applied to the set of candidate models described in Tables 5 and 6 .

We then arbitrarily accepted models for which the probability of measuring a value of $\chi^{2}$ greater than the $\chi^{2}$ calculated for the model was better than $70 \%$ for both the subgiant branch fit and the main sequence fit. In this case, only three of the trials yielded an acceptable solution. Two of the three yielded unique solutions which were not the input star formation history, but were only different in the duration of the young burst (0.5 Gyr instead of the input $1 \mathrm{Gyr})$ and the strength of the intermediate-age and young bursts $(50 \%$ and $40 \%$ respectively as opposed to the input $60 \%$ and $30 \%$ ). The third produced two solutions, one of which was the input star formation history, and one which differed only in that the intermediate-age burst was off-set by 0.5 Gyr. We therefore estimate that we can, in principle, determine the episode strength to within $10 \%$ and the episode center and duration within $0.5 \mathrm{Gyr}$, insofar as the true model is contained within the set of models which we generated. Our preliminary analysis has already led us to a reasonable range of models.

It is not entirely suprising that some of the 10 trial models were not matched by any of the 180 candidate models, despite the trial models being drawn from the candidate models. We are placing great demands on a relatively small number of stars in our dataset. Lowering the criteria would guarantee a match each time, but would increase the number of false matches. An acceptable fit can occur for two reasons - the data were truly produced by one of the input star formation history covered by the candidate models, or the data were produced by some other star formation history and an acceptable fit resulted because of stochastic effects. The cutoff criteria were set at a high percentage to eliminate as many "false" matches as possible. If the criteria resulted in no correct matches for our Carina data, we would then be forced to lower the criteria, and eventually at some point to consider a new set of candidate models. This last possibility would probably not be necessary given that we chose the set of models based on a preliminary analysis of the Carina CMD. 


\section{Results}

Having established that the selection criteria in the previous section worked reasonably well, we applied the same criteria to the 180 large models generated for comparison with our data. The best fit models, those with the probability of $c h i^{2}$ for both the main sequence and subgiant branch fits better than $70 \%$, are shown in Table 7 . The main sequence and subgiant branch $\chi^{2}$ probabilities for each model were averaged, and this value was used to rank the models in this subset. In addition, models with the probability of $c h i^{2}$ for both the main sequence and subgiant branch fits better than $50 \%$ are also listed. The individual models are not as important as the general properties of models which passed the selection criteria. These characteristics are summarized in Table 8, and examined in detail below.

In order to fully demonstrate the goodness of the best fits, we show in Figure 16 pseudo-LFs for three models which did not pass the cut. Discrepancy exists both in the normalization and in the shape of the pseudo-LFs. Recall that due to the way in which we define the pseudo-LFs, the normalization is not a free parameter; the pseudo-LFs cannot be shifted vertically at all in Figure 16. Qualitative discrepancy is found in the pseudo-LFs of all three models. Some of the effects are systematic in nature, i.e. related to variations in the parameters, and others are statistical, i.e. related to the stochastic noise in the models. The latter should be relatively small since the models are 10 times as large as the data.

In contrast, the three best fitting models are compared in Figure 17, which shows Carina's CMD as well as those of the three best fit models. Note that the three CMDs resemble each other and the data with the exception of somewhat too many stars in the Hertzprung gap at 3 and 7 Gyr. This could either represent an overestimate of the size of those populations in our models, or reflect a problem with the physics of the stellar models during that phase. Similarly, the main sequence and subgiant branch fits (Figure 18) are very good. Not only the overall shape but also the normalization is correct. Because the

models are 10 times as large as the dataset, the models are less noisy at the faint end in the MS pseudo-LF than the data, and are thus smooth where the data are spiky. The mean trends, however, are the same in these models as in the data.

Some properties of the successful models are compared in Table 8. An important episode of star formation 7 Gyr ago is one common property of all of the models listed in table 7 . Table 8 shows that 10 of the 12 models had $t_{2}=7 \mathrm{Gyr}$ and the remaining two had $t_{2}=6.5$ Gyr. However, the intermediate-age peak in the subgiant branch pseudo-LF for the models with $t_{2}=6.5 \mathrm{Gyr}$ is consistently too bright (Figure 19). No model survived which had $t_{2}=8$ Gyr. Thus $t_{2}$ is well-constrained to a narrow range around 7 Gyr. Table 8 also shows that no value of $\Delta_{2}$ can be conclusively ruled out. Given these results, we were concerned that our choices for $\Delta_{2}$ may not have bracketed the true value. As a test, 
we generated a model with the same parameters as one of the three best fit models, but $\Delta_{2}$ $=3$ Gyr. It is apparent in Figure 20 that the resulting intermediate-age peak is now too broad. The photometric errors make an episode shorter than 0.5 Gyr impossible to resolve.

The intermediate-age episode is confirmed as the dominant epoch of star formation in Carina. Only models with the intermediate-age episode stronger than the young episode by at least $20 \%$ survived the selection criteria. Although exact age information about the old population was out of reach, we were successful in constraining the strength of the old population, $S_{3}$, which was expected to mainly affect the normalization of the main sequence pseudo-LF. Table 8 shows that models with $S_{3}=0.2$ were far more likely to produce a good fit than those with $S_{3}=0.1$ or 0.3 .

Detailed information about the 3 Gyr episode of star formation was elusive, as expected. The age of the episode is constrained by the magnitude of the corresponding subgiant branch peak. But models with an episode lasting 0.5 Gyr were just as likely to produce a reasonable fit as those with star formation lasting $1 \mathrm{Gyr}$. It is possible that either our choices for $\Delta_{1}$ were not near to the true value, or our method was somehow not sensitive to changes in this parameter. As this youngest population accounts for $30 \%$ or less of the total, and because the mass function and evolution conspire to depopulate this region, there was a relatively small number of stars with which to work. Finally, we point out that there is already evidence of an even younger population ( $\leq 3 \mathrm{Gyr}$ ) in Carina, as shown in Smecker-Hane et al. 1996. However, we have too few stars to constrain such a weak, young population.

\section{Conclusion}

Carina experienced an episode of star formation 7 Gyr ago which lasted no more than 2 Gyr and which was responsible for at least $50 \%$ of its stars. The old population $(12-15$ Gyr) may amount to $10 \%-20 \%$ of Carina. The bulk of the remainder $((20 \%-30 \%)$ is relatively young, between 2.5 and 3.5 Gyr old. While the details of one galaxy's SF history may seem inconsequential, taken in context and as a member of the Local Group dwarf population, the details are relevant to deeper, unresolved issues in cosmology and galaxy evolution.

In the case of Carina, all studies confirm that at least one relatively long pause in star formation lasting $\sim 4$ Gyr occurred. The mechanism by which this kind of low mass system could experience such a pause and then another strong burst of star formation is

not understood. Current ISM simulations can produce such a large gap in an isolated dwarf 
only by ejecting the gas out to $\sim 20 \mathrm{kpc}$ (Babul 1996). A dwarf such as Carina, residing in the potential well of the Galaxy, should lose that gas, preventing any further episodes of star formation.

Recent Hubble Space Telescope observations of Carina have been studied by Mighell (1997). He claims to detect a significant number of stars in the region of the gap in star formation lasting from roughly 8 to $12 \mathrm{Gyr}$ ago. This is interpreted as indicative of significant star formation which began in the central region of Carina, and propagated to the outer regions. However, the number of stars in the same section of our CMD, derived from fields several arcminutes from the center of Carina and overlapping the Mighell (1990) field, is consistent with the number in the HST CMD. Whether or not the number of stars in this area implies significant on-going star formation, there does not seem to be evidence of a difference between the central region and regions further out, such as that seen in the recently-discovered Antlia dwarf galaxy (Aparicio et al 1997c).

Assigning ages to individual faint stars based on the isochrones which constrain them in color and magnitude must be handled with care. Firstly, at faint magnitudes, stars have a larger photometric error and thus a larger implied age range. Age determinations for specific stars are therefore subject to greater statistical uncertainty. Secondly, the lifetime of stars crossing the Hertzprung gap increases as stars become less massive. This must be accounted for when predicting the relative contributions of different ages. In light of these considerations, the results of the WFPC2 study may not differ from earlier ground based results.

Evidence of intermediate-age stars in the halo leads to the question of whether dwarf spheroidals shredded by the Galaxy could be a significant source of the halo population (Preston et al. 1994; Mateo 1996). The Sagittarius dSph is currently being ripped apart by the Milky Way, proving that this type of interaction between a large galaxy and its tiny neighbors does occur (Ibata, Gilmore, \& Irwin 1994; Mateo et al. 1995). In addition, the dSph, especially Carina and Leo I, contain significant numbers of intermediate-age stars. Mateo (1996) shows that the fraction of relatively young and intermediate-age stars in the entire population of dwarf spheroidals is not inconsistent with the fraction in the Galactic halo.

The detailed interpretation of deep galaxy counts and redshift surveys depends on the SFH of the dwarfs which comprise the excess of faint galaxies. For example, 'flashing' dwarfs - i.e. bursting quickly and intensely - contribute to the deep counts to a dramatically different extent than do dwarfs that experience more drawn-out bursts that slowly turn on and off (Campos 1997). The prevalence of bursting behavior in the SFH of Local Group dwarfs may statistically constrain the degree to which galaxies similar to these local dwarfs 
could be responsible for the faint excess.

We can place an upper limit on the star formation rate (SFR) of Carina 7 Gyr ago by assuming that the episode of star formation took place instantaneously. By choosing a reasonable model, we can easily calculate the total number of stars formed during this episode in the volume of the galaxy covered by our three fields, taking into account the incompleteness. We can use this number to normalize the IMF, and then integrate to get the total mass formed during this episode. The SFR is then given by:

$$
S F R=\frac{M_{t o t a l}}{\Delta t} * \frac{1}{\gamma} M_{\odot} y r^{-1}
$$

where $\Delta t$ is the duration of the episode in years and $\gamma$ is the fraction of the galaxy's total surface brightness in the three fields. If the episode lasted $10 \mathrm{Myr}$, the implied SFR for Carina at that time would be roughly $0.8 M_{\odot} y r^{-1}$. If the episode lasted 2 Gyr, the SFR is down by more than a factor of 100 to $0.004 M_{\odot} y r^{-1}$. Either SFR is comparable to the instantaneous SFR in even such luminous objects as blue compact dwarfs (Fanelli et al. 1988).

Could Carina-type galaxies contribute to the excess of faint galaxies at around $B \sim 24$ ? These galaxies have been shown to have redshifts between 0.3 and 0.7 (Glazebrook et al. 1995). Assuming $H_{\circ}=50 \mathrm{~km} / \mathrm{s} / \mathrm{Mpc}$, and $\Omega=1,7$ Gyr corresponds to $z \sim 0.5$, which places the episode of star formation in the appropriate epoch. If the burst were essentially instantaneous, the implied total luminosity of Carina at 7 Gyr would be $\sim 2 \times 10^{8} L_{\odot}$. Carina would be visible to $z \sim 0.3$ in a sample limited to $V \sim 25$, putting it at the near edge of objects that could contribute to these counts. Galaxies 3-10 times more luminous would fall in the range $z \sim 0.5$ to 1.0. Although the local dSph cover a range up to 50 times the luminosity of Carina, Carina is the most extreme example of this type of episodic behavior. Further, if the episodes of star formation are extended in time, then these dwarfs would only be visible locally. Thus, despite concerted efforts at unravelling the SFH of these galaxies, their role in faint galaxy counts problem remains unresolved.

\section{Acknowledgements}

The authors would like to thank Antonio Aparicio for his thoughtful comments regarding this paper. DHK thanks Philipe Fischer and Andrew Layden for their helpful comments, as well. This research was funded in part by NSF. 


\section{REFERENCES}

Aparicio, A., Gallart, C., \& Bertelli, G. 1997a, AJ, 114, 680

Aparicio, A., Gallart, C., \& Bertelli, G. 1997b, AJ, 114, 669

Aparicio, A., Delcanton, J., Gallart, C., \& Martinez-Delgado, D. 1997c, AJ, 114, 1447

Babul, A. 1996, private communication

Bertelli, G., Mateo, M., Chiosi, C., \& Bressan, A. 1992, ApJ, 388, 400

Bergbusch, P. A., \& VandenBerg, D. A. 1992, ApJS, 81, 163

Campos, A. 1997, ApJ, 488, 606

Cannon, R.D., Niss, B., \& Norgaard-Nielsen, H. U. 1981, MNRAS, 196, 1P

Da Costa, G. S. 1994, ESO/OHP Workshop on Dwarf Galaxies, eds. G. Meylan \& P. Prugniel, p. 221

Da Costa, G. S., \& Armandroff, T. E. 1990, AJ, 100, 162.

Da Costa, G. S., Armandroff, T. E., Caldwell, N., Seitzer, P. 1996, AJ, 112, 2576

Fanelli, M., O’Connell, R., \& Thuan, T. 1988, ApJ, 334, 665

Ferguson, H. 1996, private communication

Flannery, B., \& Johnson, B. 1982, ApJ, 263, 166

Fusi Pecci, F., Ferraro, F., Crocker, D., \& Buonanno, R. 1990, A\&A, 238, 95

Fusi Pecci, F., Ferraro, F., Corsi, C., Cacciari, C., \& Buonanno, R. 1992, AJ, 104, 1831

Gallart, C., Aparicio, A., Bertelli, G., \& Chiosi, C. 1996a, AJ, 112, 1950

Gallart, C., Aparicio, A., Bertelli, G., \& Chiosi, C. 1996b, AJ, 112, 2596

Glazebrook, K., Ellis, R., Colless, M., Broadhurst, T., Allington-Smith, J., \& Tanvir, N. 1995, MNRAS, 273, 157

Graham, J. A. 1982, PASP, 94, 244

Lee, Y., Demarque, P., \& Zinn, R. 1990, ApJ, 350, 155

Hunter, D. A., Light, R. M., Holtzman, J. A., Lynds, R., O’Neil, E. J., Jr., \& Grillmair, C. J. 1997, ApJ, 478, 124

Ibata, R. A., Gilmore, G., \& Irwin, M. J. 1994, Nature, 370, 194

Mateo, M. 1988, ApJ, 331, 261

Mateo, M., Nemec, J., Irwin, M., \& McMahon, R. 1991, AJ, 101, 892 
Mateo, M., Udalski, A., Szymanski,M., Kaluzny, J., Kubiak, M., \& Krzeminski, W. 1995, AJ, 109, 588

Mateo, M. 1996, Formation of the Galactic Halo...Inside and Out, Heather Morrison and Ata Sarajedini, eds., ASP Conference Series, Vol. 92 1996, p. 434

Mateo, M., Hurley-Keller, D. \& Nemec, J. 1998, AJ, submitted

Mighell, K. J. 1990, A\&AS, 82, 1

Mighell, K. J. \& Butcher, H. R. 1992, A\&A, 255, 26

Mighell, K. J. 1997, AJ, 114, 1458

Mould, J. R. \& Aaronson, M. 1983, ApJ, 273, 530

Preston, G. W., Beers, T. C., \& Shectman, S. A. 1994, AJ, 108, 538

Paczynski, B. 1984, ApJ, 284, 670

Ratcliff, S. 1987, ApJ, 318, 196

Saha, A., Monet, D. G., \& Seitzer, P. AJ, 1986, 92, 302

Salpeter, E. E. 1955, ApJ, 121, 161

Sandage, A. 1982, ApJ, 252, 553

Sarajedini, A. \& Da Costa, G. S. 1991, AJ, 102, 628

Schechter, P., Mateo, M., \& Saha, A., 1993, PASP, 105, 1342

Smecker-Hane, T. A., Stetson, P. B., Hesser, J. E., \& Lehnert, M. D. 1994, AJ, 108, 507

Smecker-Hane, T. A., Stetson, P. B., Hesser, J. E., \& VandenBerg, D.A. 1996, From Stars to Galaxies: The Impact of Stellar Physics on Galaxy Evolution, eds C. Leitherer, U. Fritze-von Alvensleben, and J Huchra (ASP: San Francisco), p. 328.

Tolstoy, E. 1996, ApJ, 462, 684

Tosi, M., Greggio, L., Marconi, G., \& Focardi, P. 1991, AJ, 102, 951

Trippico, M., Dorman, B. \& Bell 1993, AJ, 106, 618

Vallenari, A., Aparicio, A., Fagotto, F., \& Chiosi, C. 1994, A\&A, 284, 424

Vallenari, A., Chiosi, C., Bertelli, G., Aparicio, A., \& Ortolani, S. 1996, A\&A, 309, 367

VandenBerg, D. A. 1983, ApJS, 51, 29

VandenBerg, D. A. 1992, ApJ, 391, 685 
Fig. 1. - The Carina dwarf galaxy. Outlined are the three fields discussed in this paper, labelled F1, F2, and F3. Each field is $3.89^{\prime}$ across. Also, the field observed by Mighell (1990) is labelled M. The orientation is North to the top and West to the right.

Fig. 2.- Error in $V$ magnitude vs. $V$ magnitude.

Fig. 3.- Error in $B-V$ color vs. $V$ magnitude.

Fig. 4.- Results of the false star analysis which shows the difference between the input magnitude, $m_{i}$, and the measured magnitude, $m_{o}$, vs. $m_{i}$ for field 1 .

Fig. 5.- Combined completeness factors as a function of $V$ magnitude and $B-V$ color for the three Carina fields.

Fig. 6. - Color-magnitude diagrams for the three fields observed in Carina. (a) Field 1, (b) Field 2, (c) Field 3, (d) All three fields combined. The solid line represents the limiting magnitude of the shallowest field, field 3 .

Fig. 7.- Comparison between Mighell's (1990) $V$ photometry and ours. $V$ represents the magnitudes presented in this paper, $V_{M}$ those of Mighell (1990). Our results are systematically 0.034 magnitudes brighter than those of Mighell (1990). This discrepancy, if real, is unlikely to affect the results of this study.

Fig. 8.- A comparison of luminosity functions. The short dashed line is derived from Mighell's data, the long dashed line from MA (1983), and the solid line from ours. No corrections have been made for completeness or contamination in any of the datasets. The observations were normalized to the number of stars in the six brightest bins.

Fig. 9.- The luminosity functions for the three fields in Carina. No correction has been made for foreground or background contamination in these luminosity functions.

Fig. 10.- Pseudo-LF bins in the main sequence and subgiant branch regions of the CMD. Each bin on the main sequence contains 50 stars, and each bin on the subgiant branch contains 30 .

Fig. 11.- Pseudo-LFs for the Carina data, R, vs. the V magnitude of the bin center. Shown are the main-sequence pseudo-LF (bottom) and the subgiant branch pseudo-LF (top).

Fig. 12.- Comparison of an interpolated isochrone with a model isochrone provided by M. Trippico. Solid line: model isochrone with age $=4 \mathrm{Gyr}$ and $[\mathrm{Fe} / \mathrm{H}]=-2.23$. Dashed line: isochrone with the same age and metallicity interpolated by the program which generates synthetic CMDs. 
Fig. 13.- The parameterization of Carina's star formation history. We assumed three major episodes of star formation. $t_{i}$ is the age of the episode in Gyr, $\Delta_{i}$ is the duration of the episode in Gyr, and $S_{i}$ is the strength of the episode in a fraction of the total population. For reasons explained in the text, $t_{1}, t_{3}$, and $\Delta_{3}$ are fixed at $3 \mathrm{Gyr}, 15 \mathrm{Gyr}$, and $1 \mathrm{Gyr}$, respectively.

Fig. 14.- The subgiant branch pseudo-LFs for four models which differ only in the value of $t_{1} . t_{2}$ is $7 \mathrm{Gyr}, \Delta_{1}$ and $\Delta_{2}$ are $1 \mathrm{Gyr}$, and $S_{1}, S_{2}$, and $S_{3}$ are $0.3,0.5$, and 0.2 , respectively. Because isochrones are relatively widely separated at magnitude $V \sim 22$, it is possible to rule out an age of $\sim 4$ Gyr for the youngest episode of star formation based on the subgiant branch pseudo-LF.

Fig. 15.- Stocastic variation between models with the same star formation history. Panel (a) shows the main sequence pseudo-LFs of five models with the same input parameters, but different random number seeds. These models have the same number of stars in the RGB region as the Carina data. Panel (b) shows the main sequence pseudo-LFs of five models with the same input parameters, but ten times as many stars in the RGB region.

Fig. 16. - Three models which fit the data poorly. Panel (a) shows the main sequence pseudo-LF and panel (b) shows the subgiant branch pseudo-LF. Solid: The Carina data. Dotted: A model with $\Delta_{1}=0.5 \mathrm{Gyr}, t_{2}=8 \mathrm{Gyr}, \Delta_{2}=0.5 \mathrm{Gyr}$, and $S_{1}, S_{2}$, and $S_{3}$ are 0.2 , 0.7 , and 0.1, respectively. Short dash: A model with $\Delta_{1}=1.0 \mathrm{Gyr}, t_{2}=6.5 \mathrm{Gyr}, \Delta_{2}=1.0$ Gyr, and $S_{1}, S_{2}$, and $S_{3}$ are $0.2,0.5$, and 0.3 , respectively. Long dash: A model with $\Delta_{1}=$ $1.0 \mathrm{Gyr}, t_{2}=7 \mathrm{Gyr}, \Delta_{2}=0.5 \mathrm{Gyr}$, and $S_{1}, S_{2}$, and $S_{3}$ are $0.1,0.7$, and 0.2 , respectively.

Fig. 17.- The color-magnitude diagrams of three models which have the best overall fit to the data. Note that control field stars have been added to these model diagrams. The Carina CMD has three times the contamination shown in the models. Panel (a) shows the combined CMD for Carina. The star formation histories for the model CMDs are shown in the remaining panels: (b) A model with $\Delta_{1}=1.0 \mathrm{Gyr}, t_{2}=7 \mathrm{Gyr}, \Delta_{2}=2.0 \mathrm{Gyr}$, and $S_{1}$, $S_{2}$, and $S_{3}$ are $0.3,0.5$, and 0.2 , respectively; (c) A model with $\Delta_{1}=0.5 \mathrm{Gyr}, t_{2}=7.0 \mathrm{Gyr}$, $\Delta_{2}=2.0 \mathrm{Gyr}$, and $S_{1}, S_{2}$, and $S_{3}$ are 0.3, 0.5, and 0.2, respectively; (d) A model with $\Delta_{1}=$ $1.0 \mathrm{Gyr}, t_{2}=7 \mathrm{Gyr}, \Delta_{2}=1.0 \mathrm{Gyr}$, and $S_{1}, S_{2}$, and $S_{3}$ are $0.3,0.5$, and 0.2 , respectively.

Fig. 18. - The pseudo-LFs of the three models which have the best overall fit to the data. Panel (a) shows the main sequence pseudo-LFs, and panel (b) the subgiant branch pseudoLFs. The sold line in each repesents the pseudo-LFs derived from the Carina data. The star formation histories for the models are explained in the caption to figure 16. 
Fig. 19.- Panel (a) shows the subgiant branch pseudo-LFs of the Carina data and three models with $t_{2}=6.5$ Gyr. These models consistently produce an intermediate-age peak ( $V \sim 22.5$ ) which is slightly too bright. Panel (b) shows the subgiant branch pseudo-LFs of the Carina data and three models with $t_{2}=8 \mathrm{Gyr}$. These models consistently produce an intermediate-age peak which is slightly too faint.

Fig. 20.- Subgiant branch pseudo-LFs of the Carina data and a model with $\Delta_{2}=3$ Gyr. This model produced an intermediate-age peak $(V \sim 22.5)$ which is clearly too broad. 


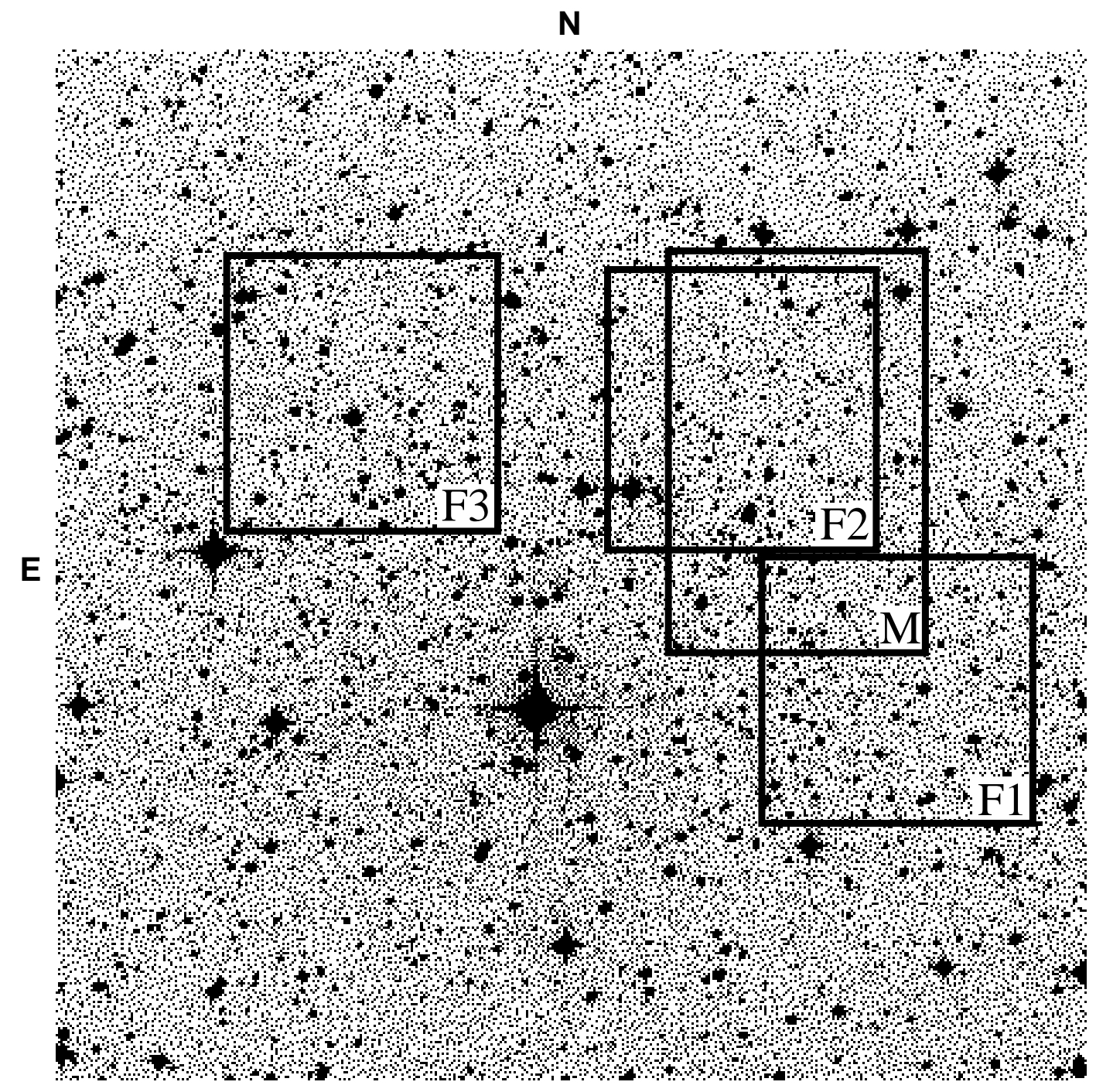

\section{Hurley-Keller et al. - Figure 1}




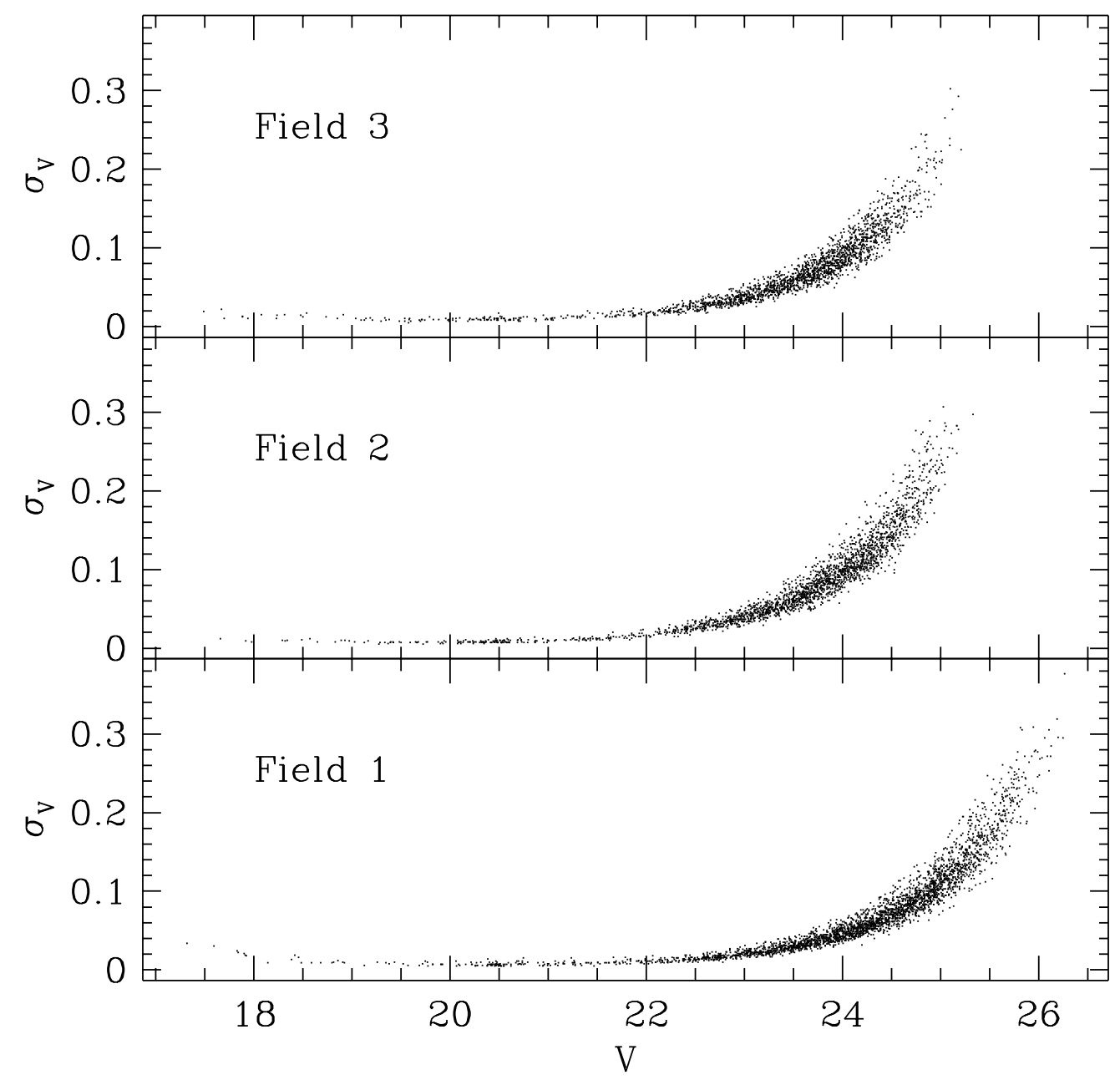

Hurley-Keller et al. - Figure 2 


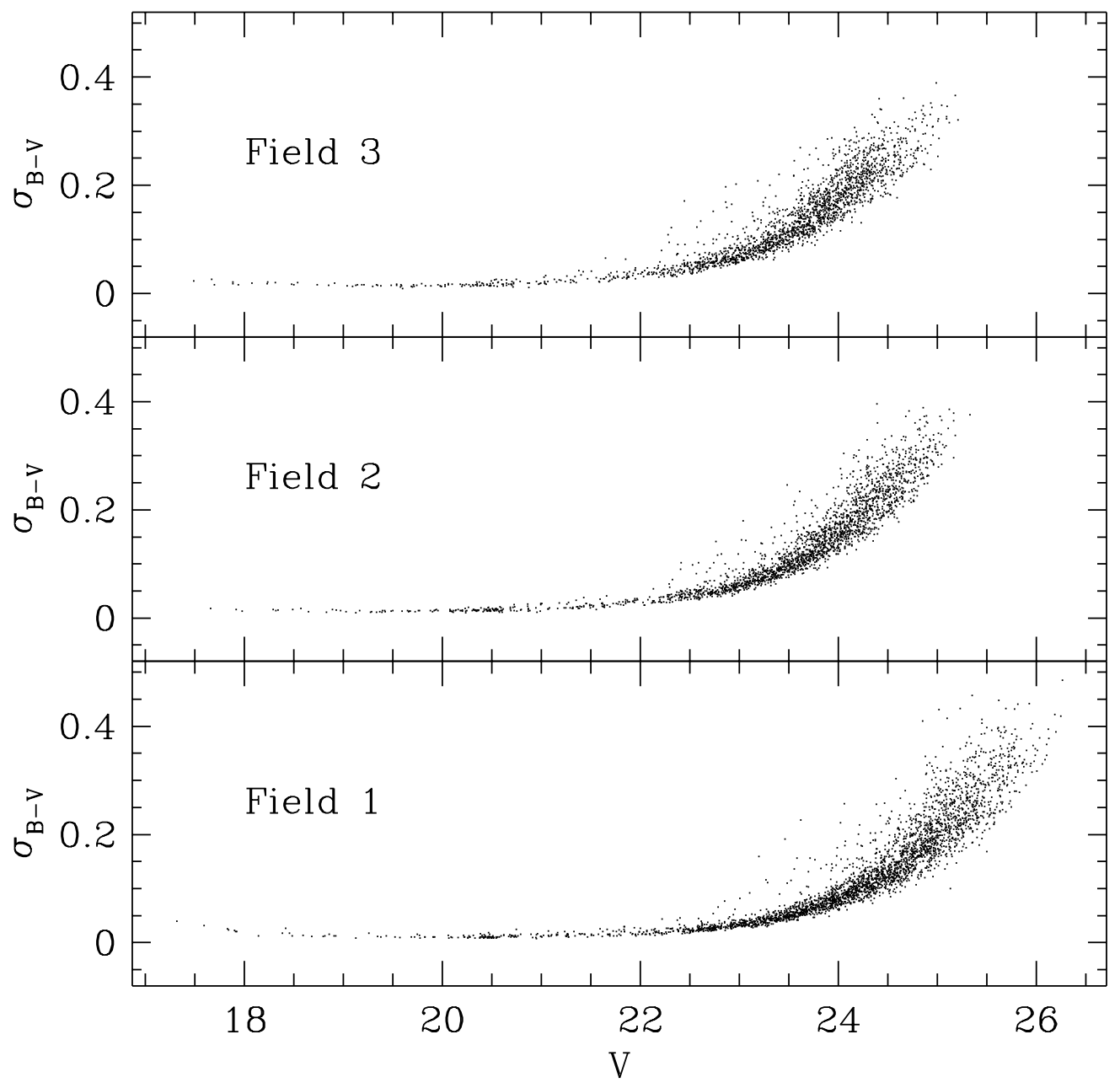

Hurley-Keller et al. - Figure 3 


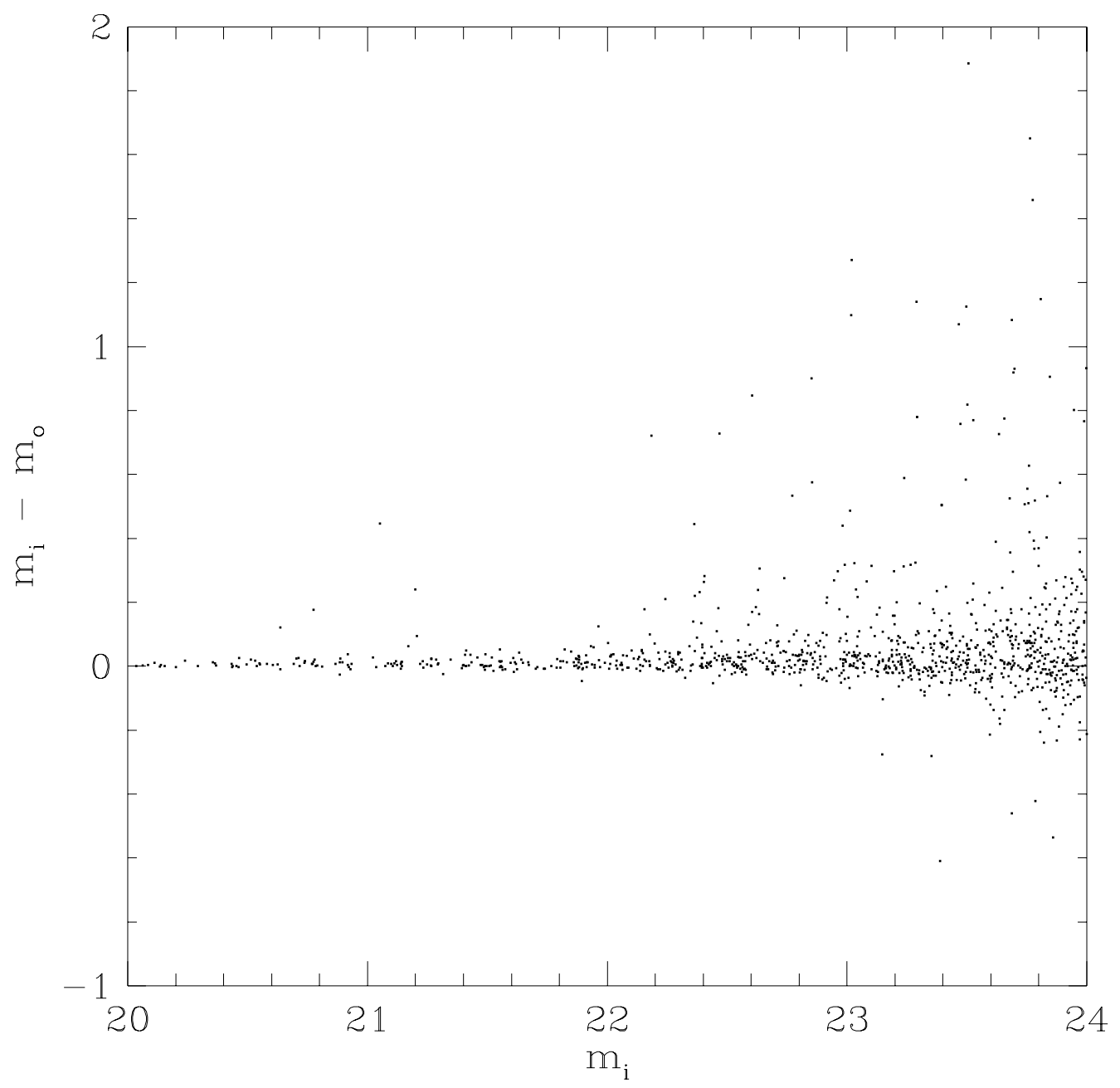

Hurley-Keller et al. - Figure 4 


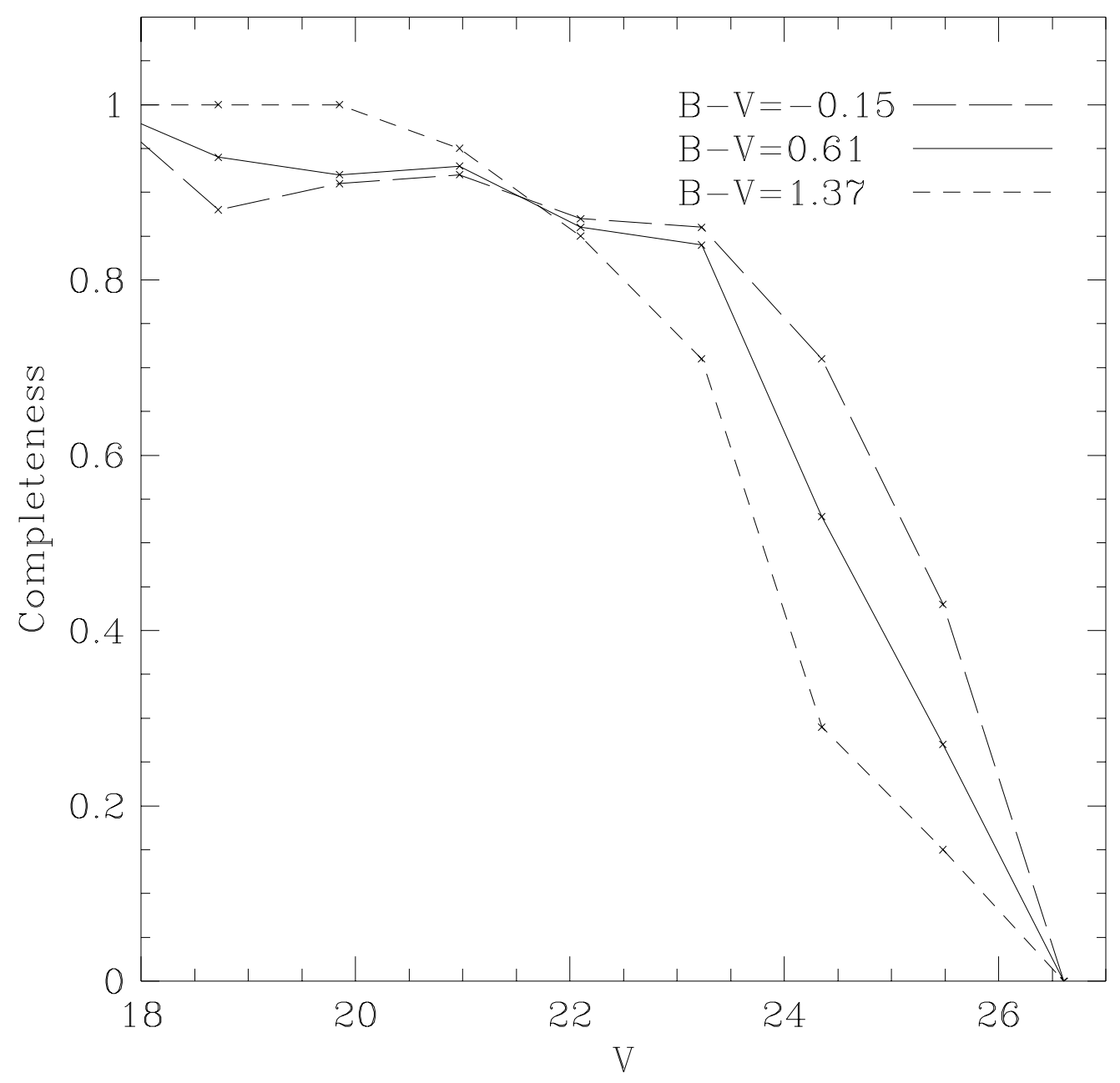

Hurley-Keller et al. - Figure 5 


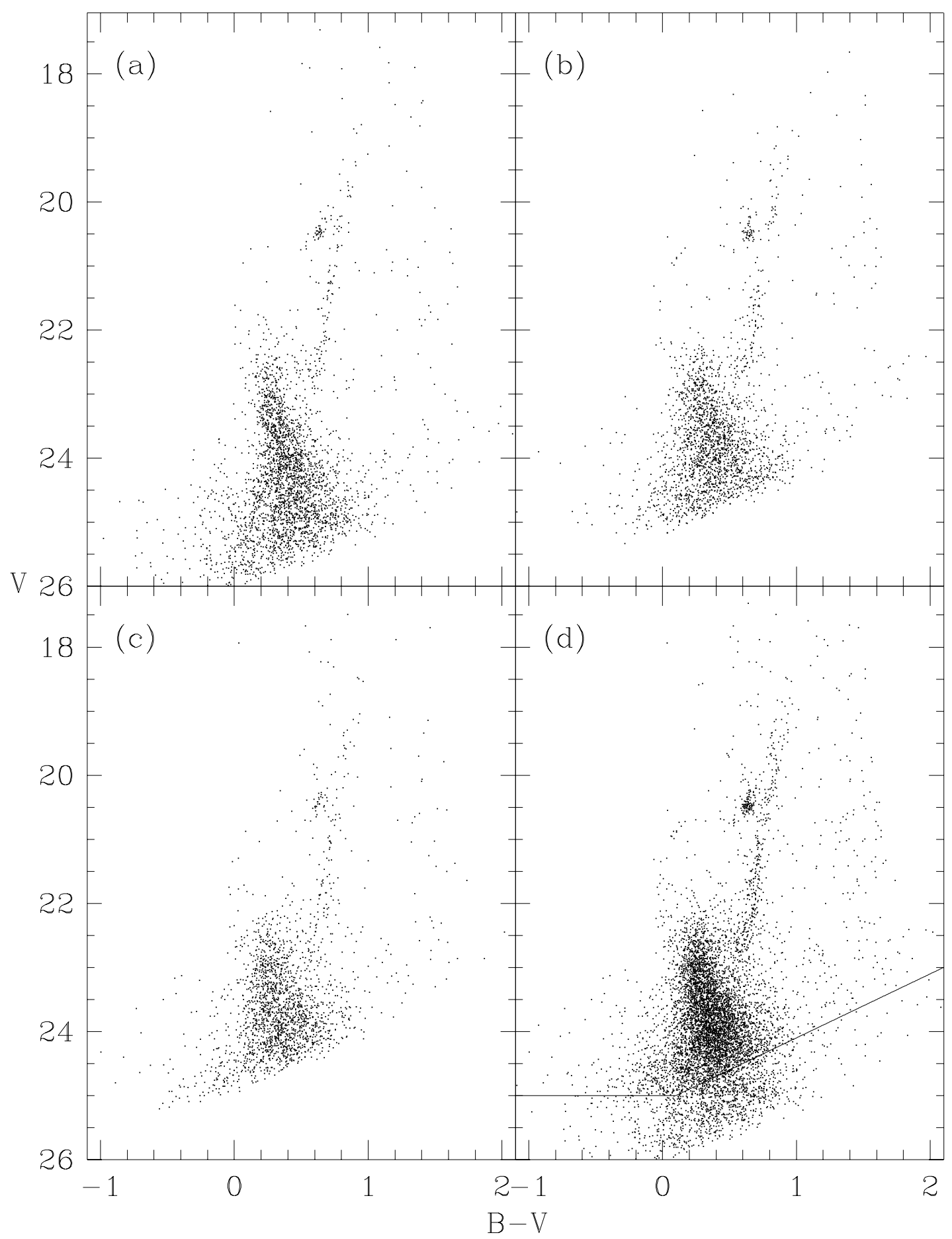

Hurley-Keller et al. - Figure 6 


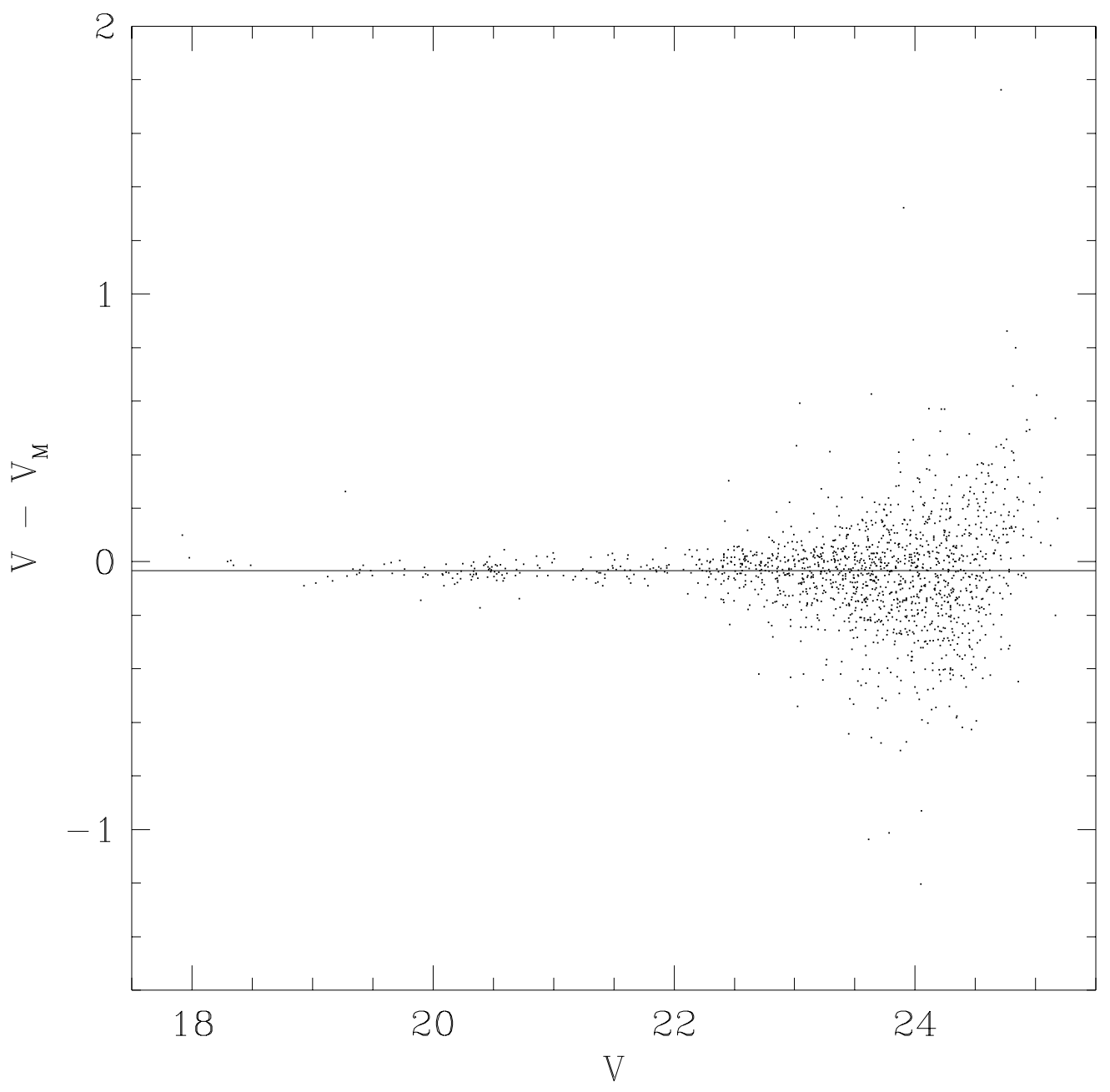

Hurley-Keller et al. - Figure ? 


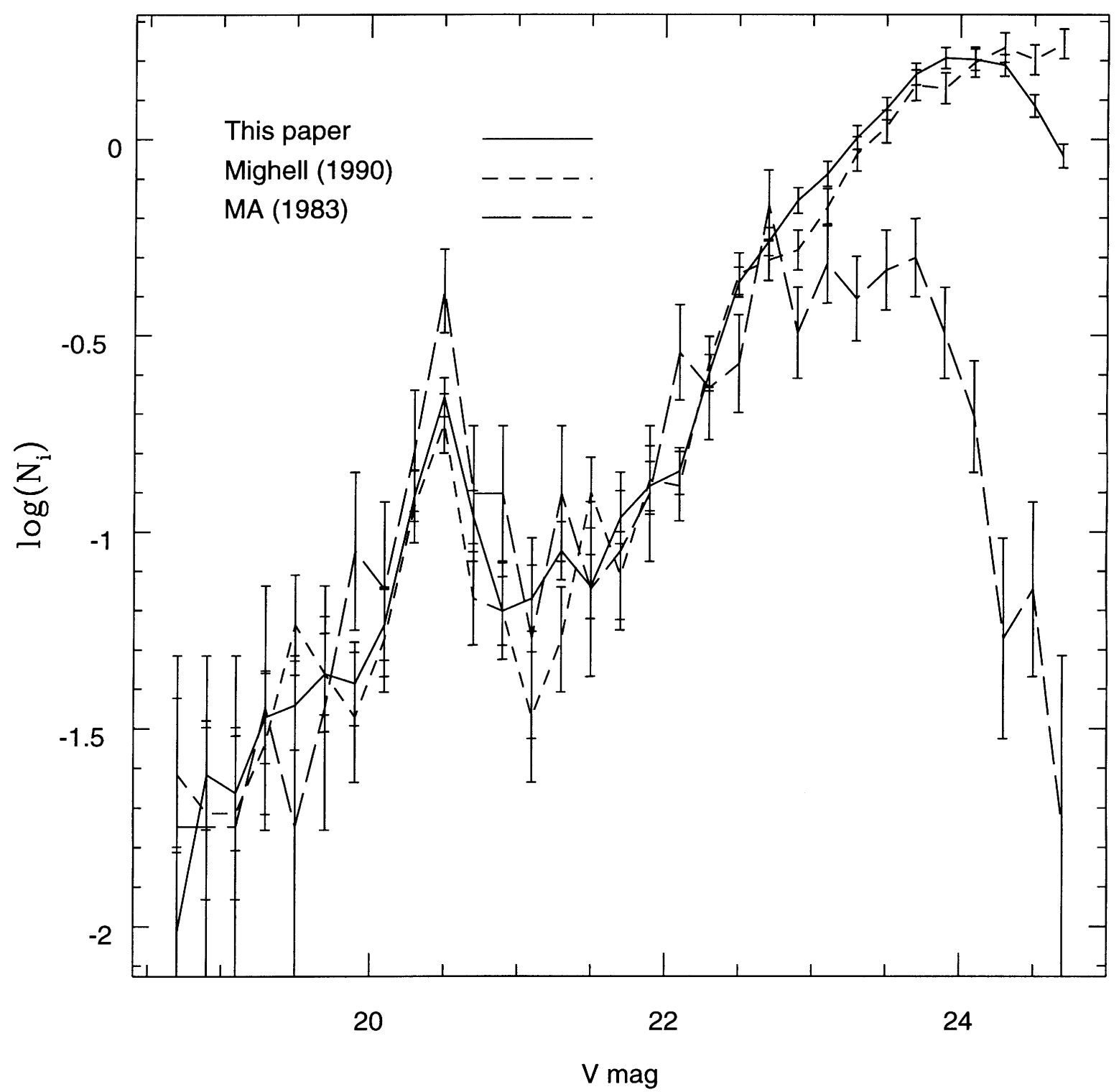

Hurley-Keller et al. - Figure 8 


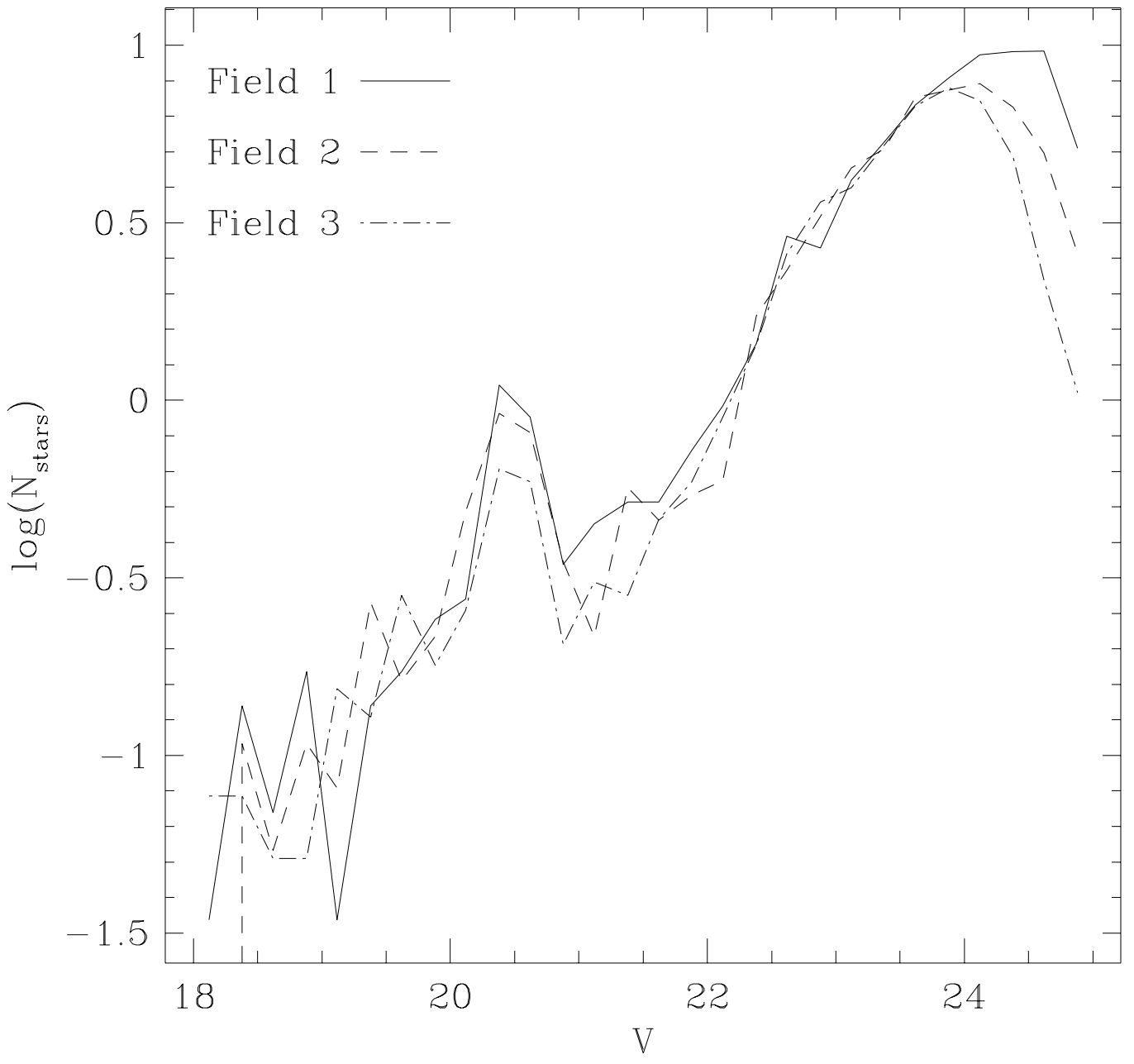

Hurley-Keller et al. - Figure 9 


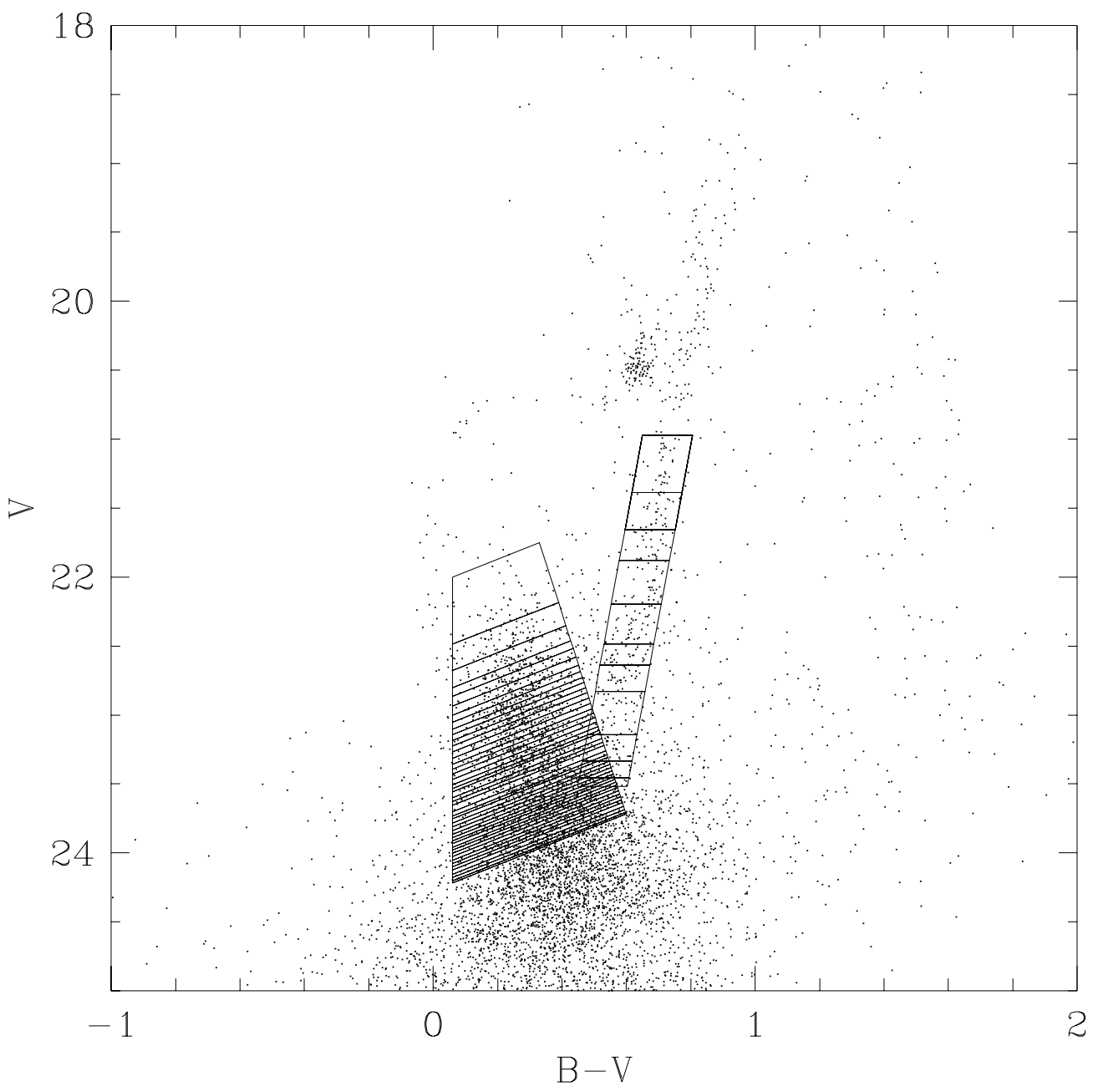

Hurley-Keller et al. - Figure 10 

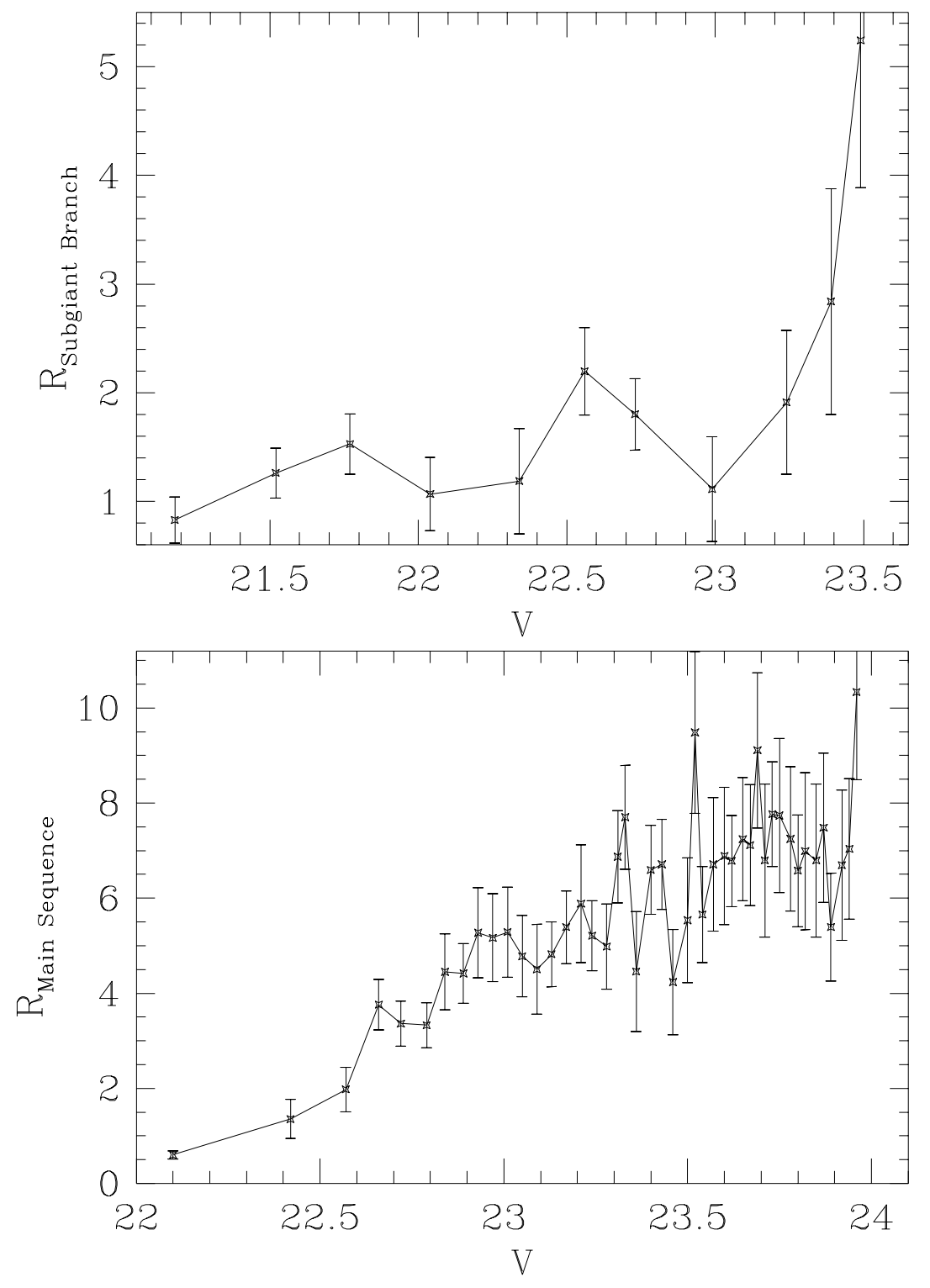

Hurley-Keller et al. - Figure 11 


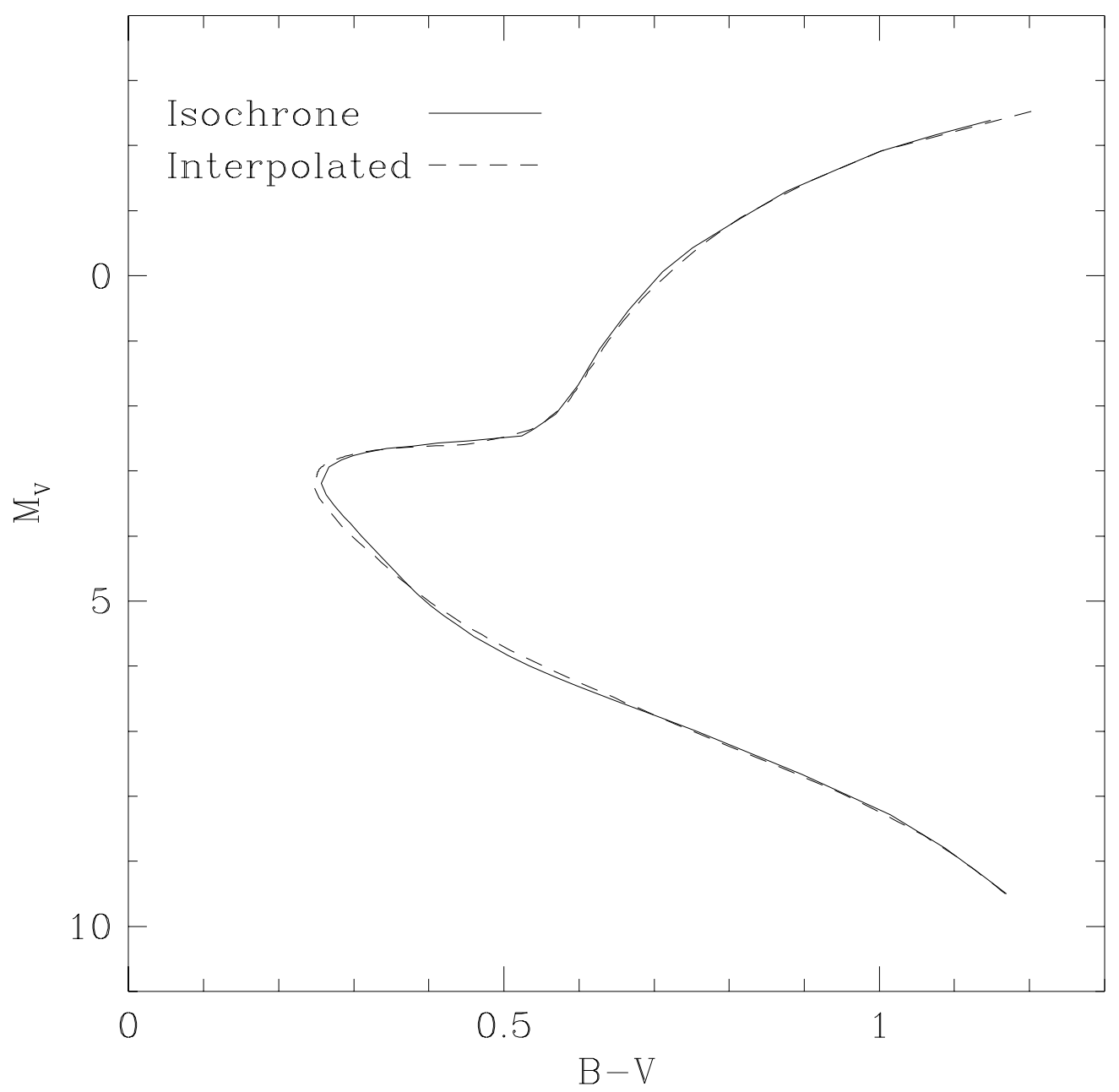

Hurley-Keller et al. - Figure 12 


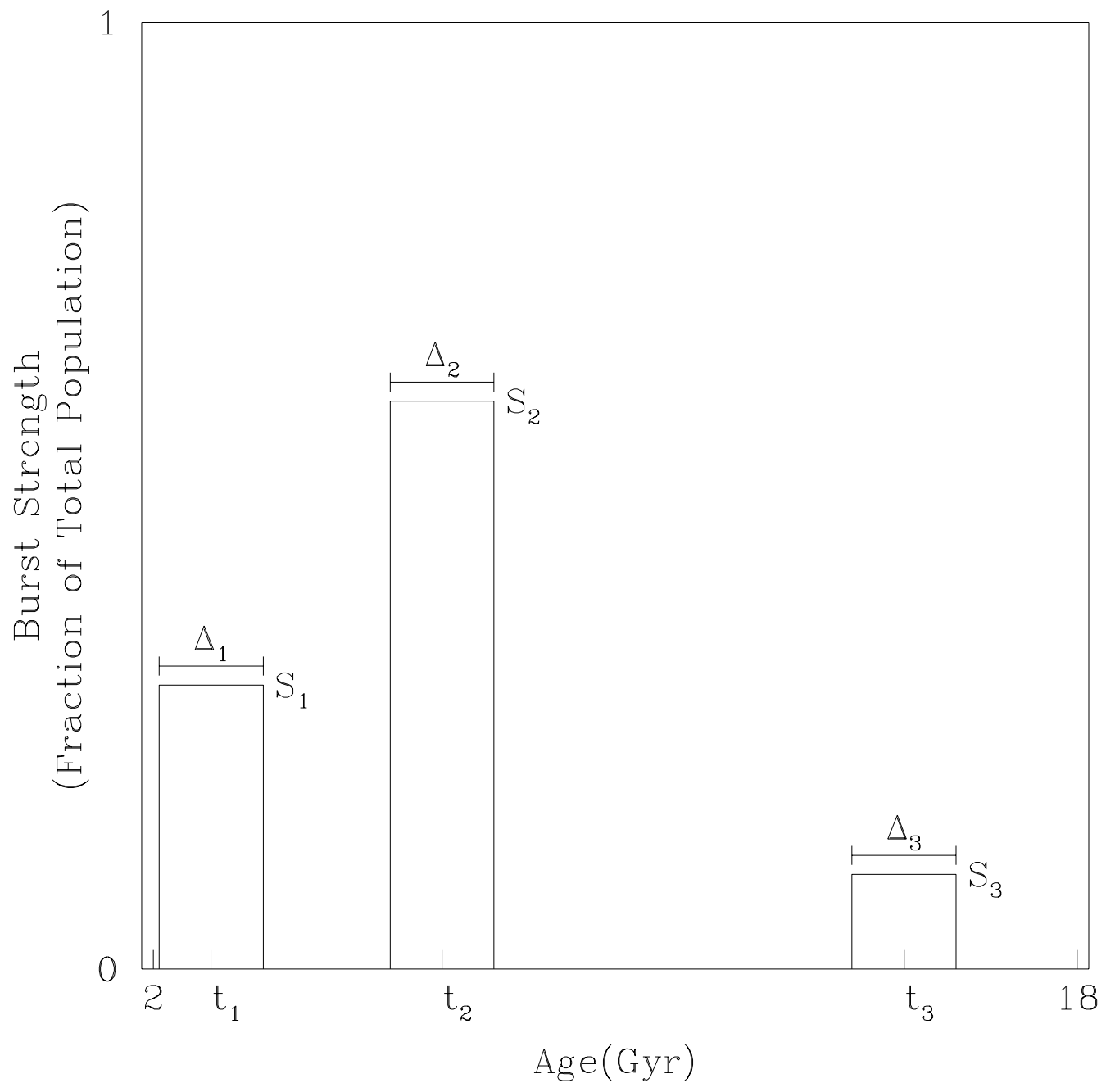

Hurley-Keller et al. - Figure 13 


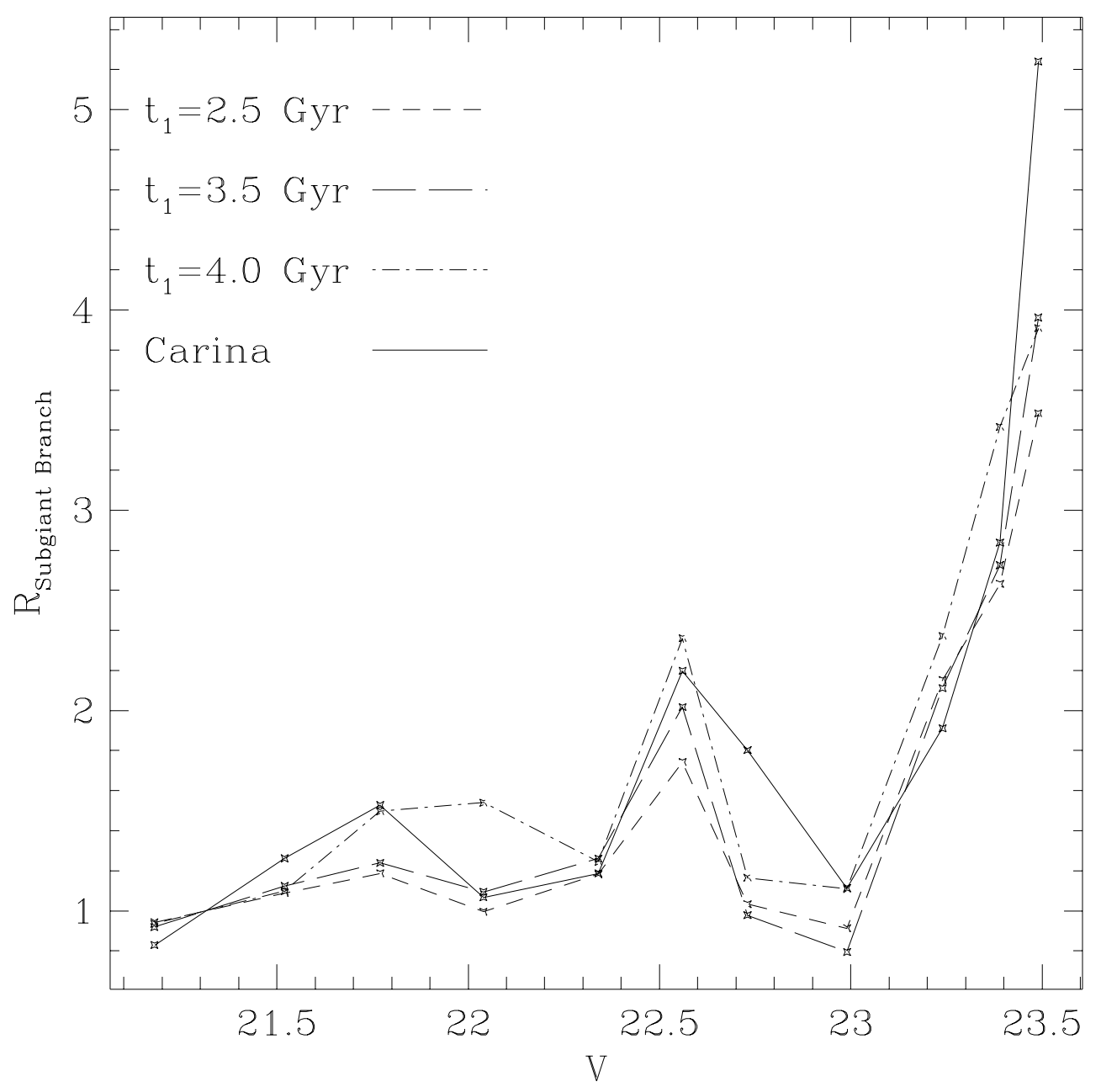

Hurley-Keller et al. - Figure 14 


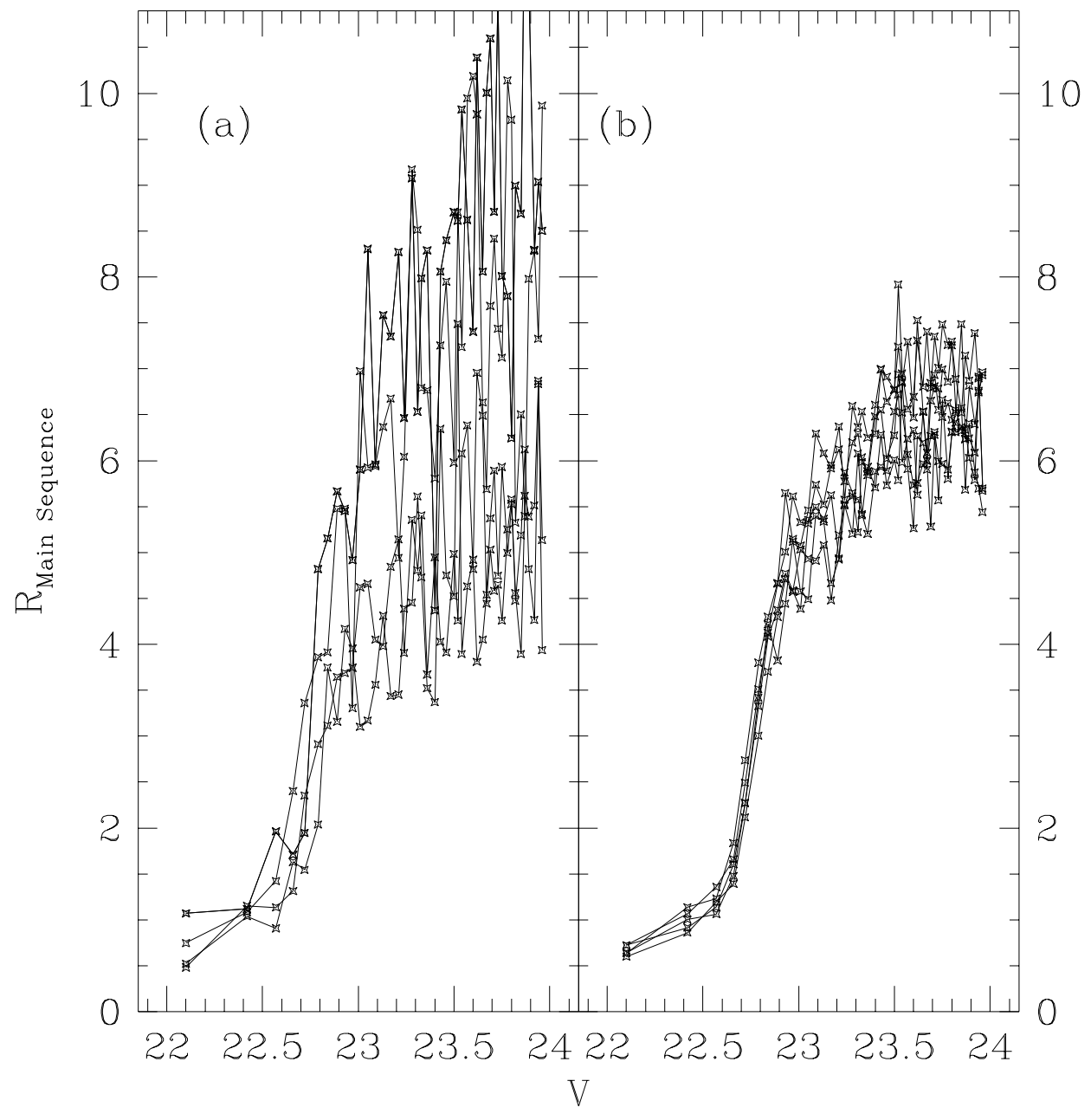

Hurley-Keller et al. - Figure 15 


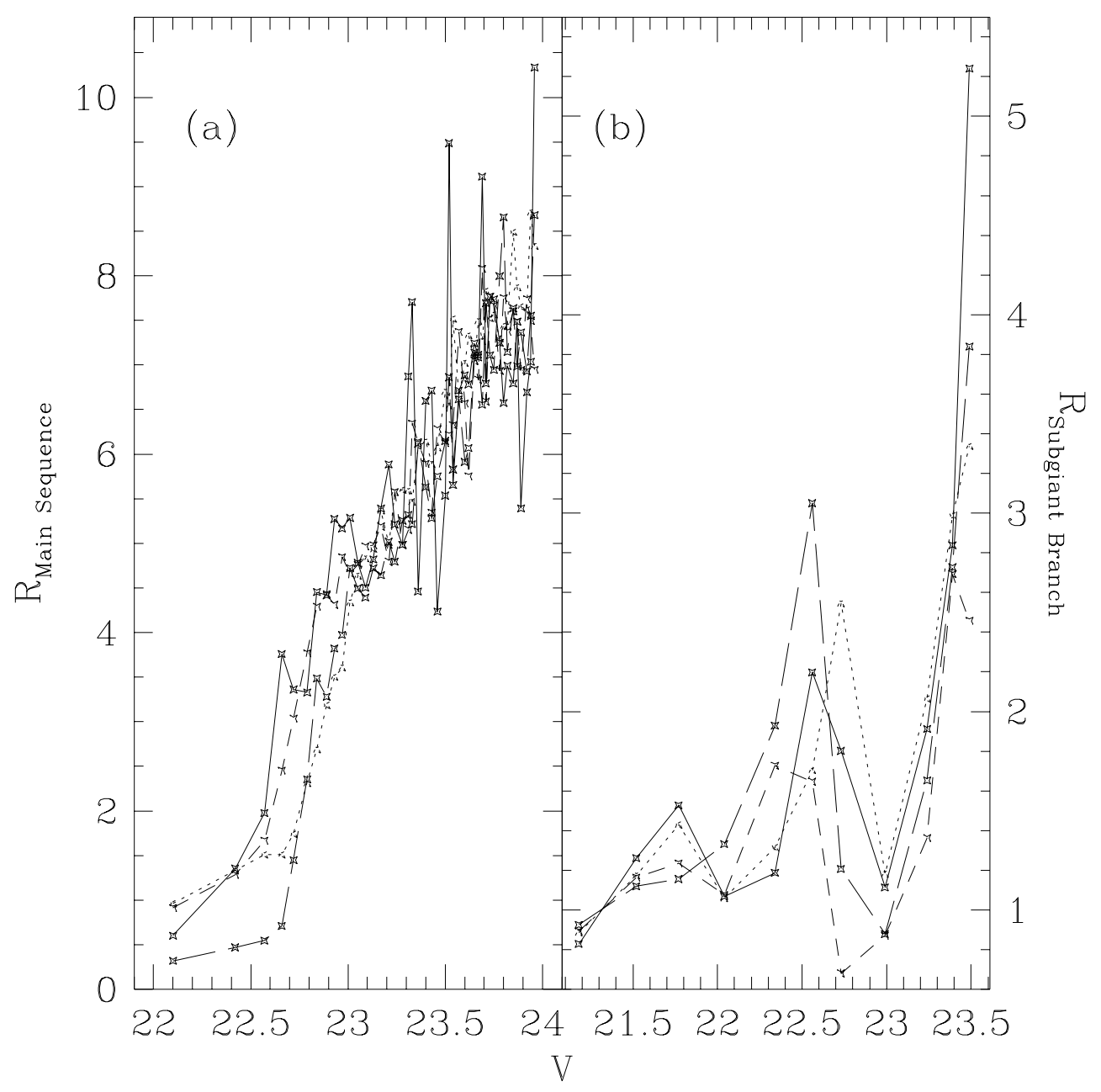

Hurley-Keller et al. - Figure 16 


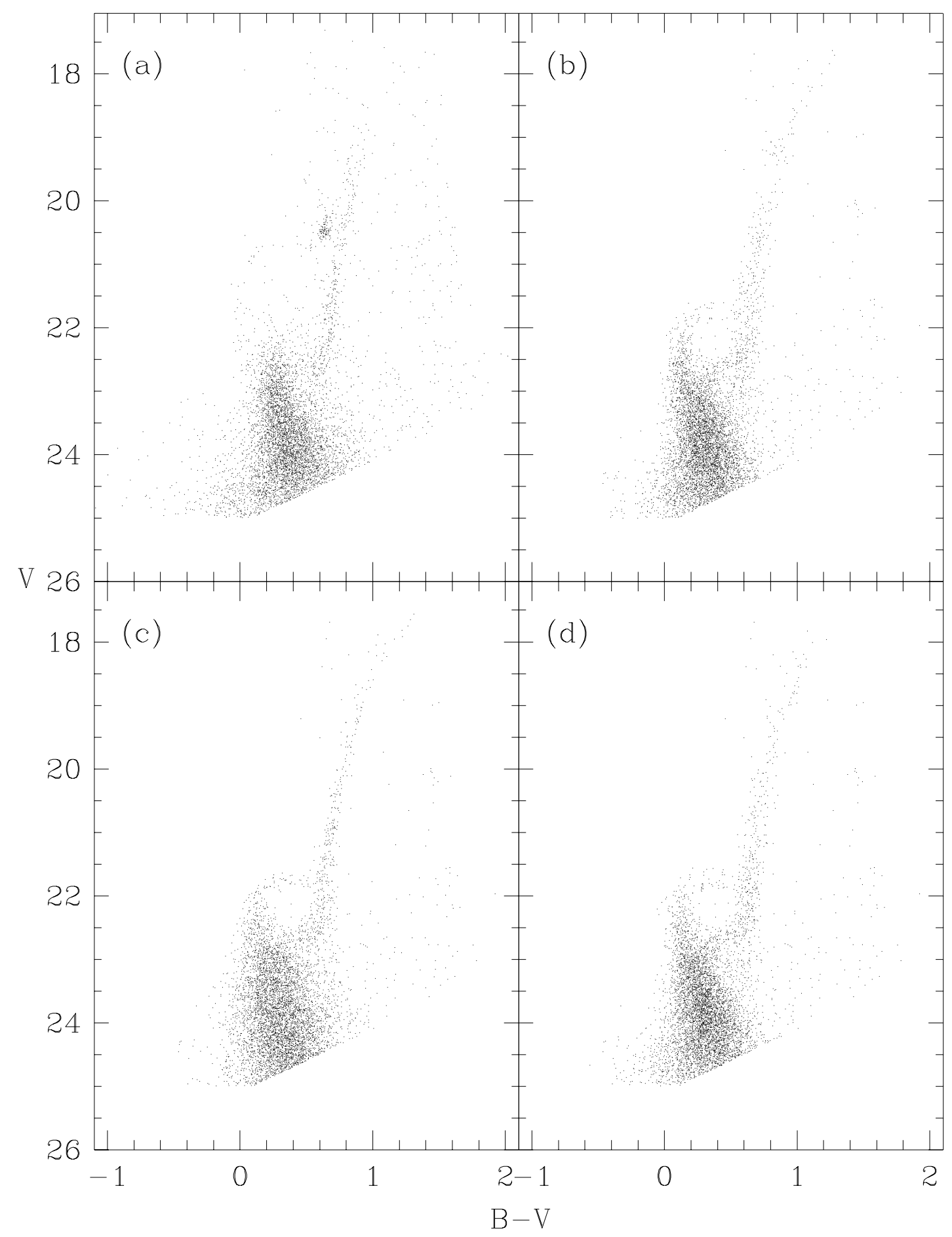

Hurley-Keller et al. - Figure 17 


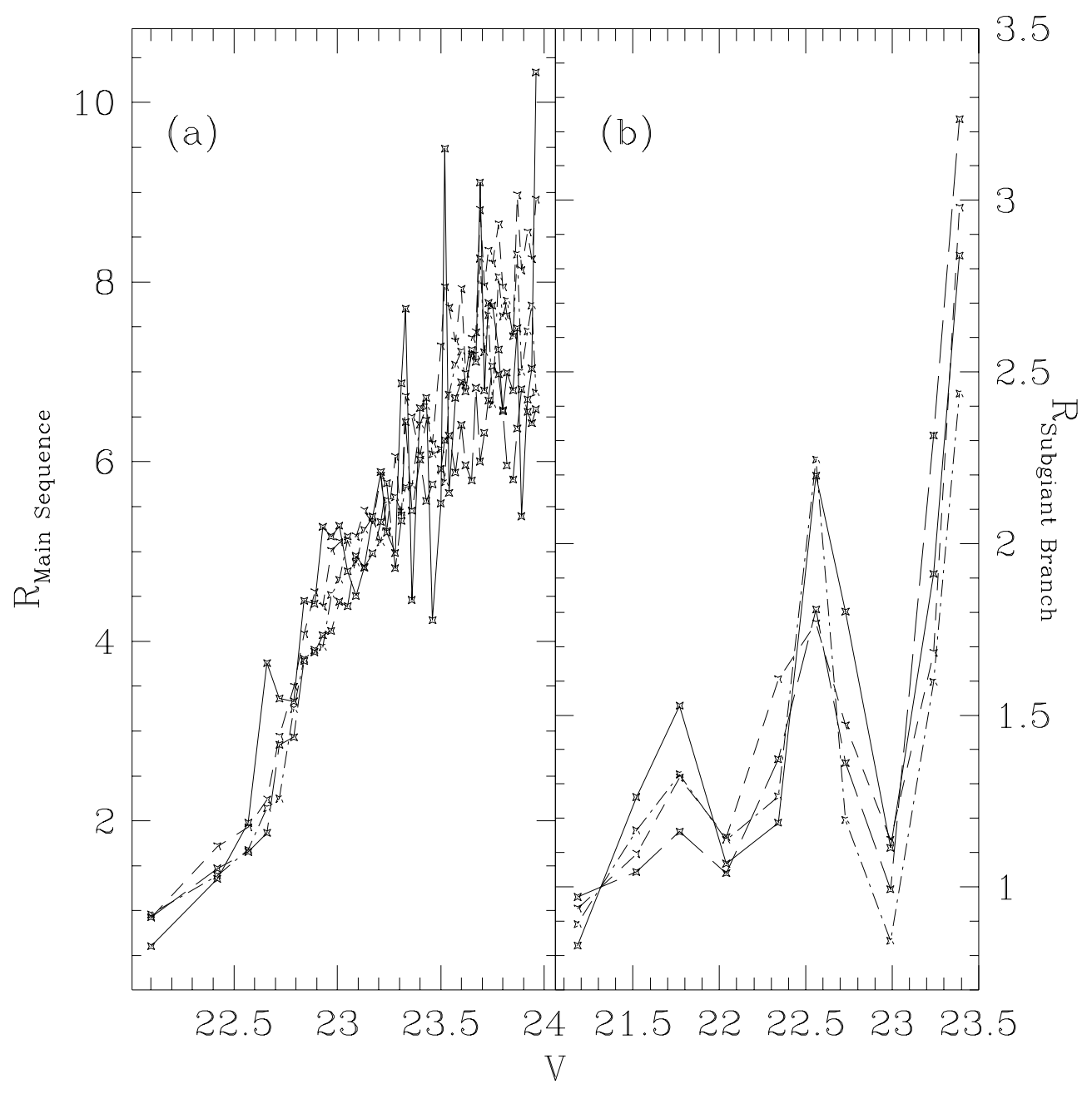

Hurley-Keller et al. - Figure 18 


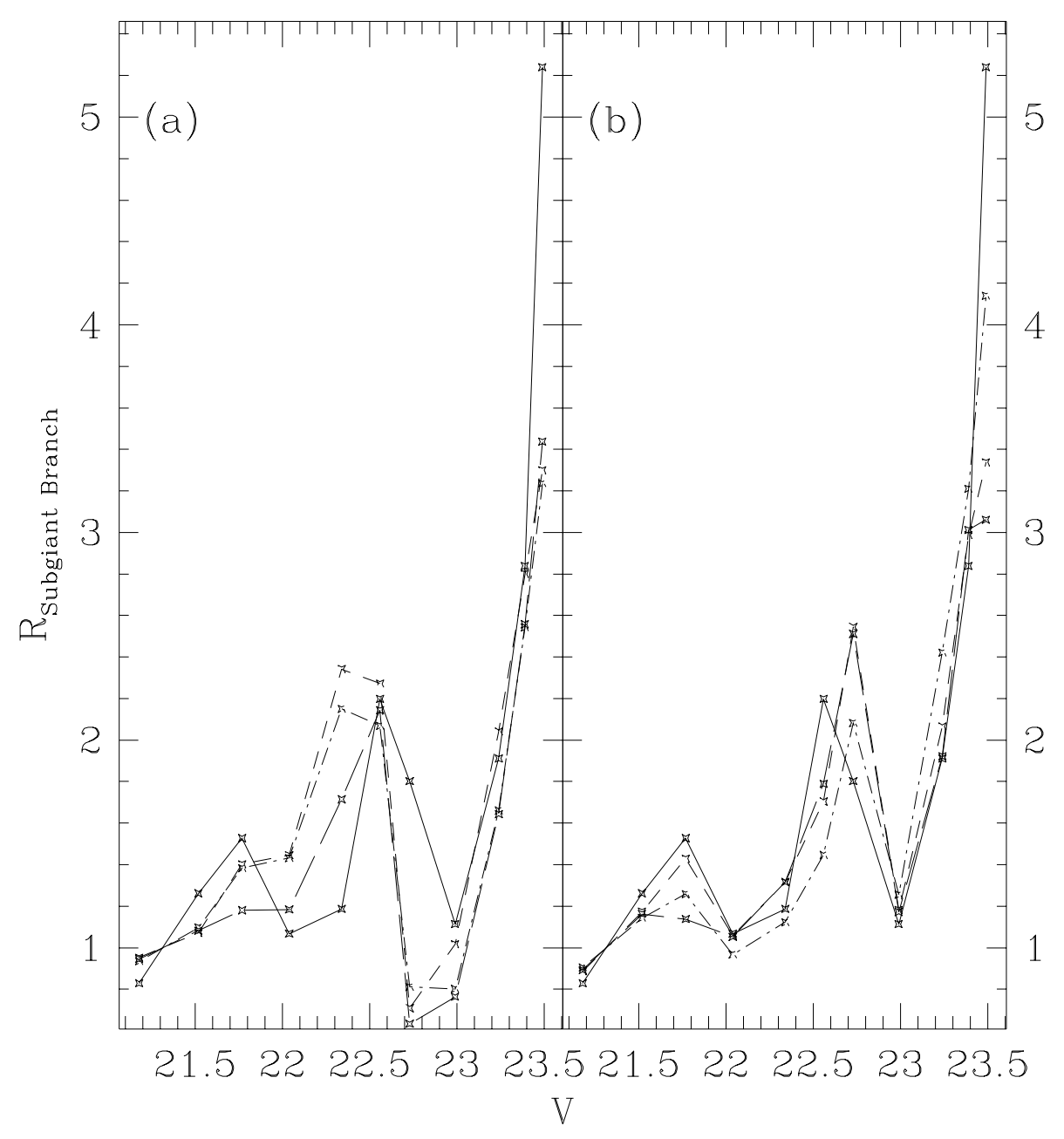

Hurley-Keller et al. - Figure 19 


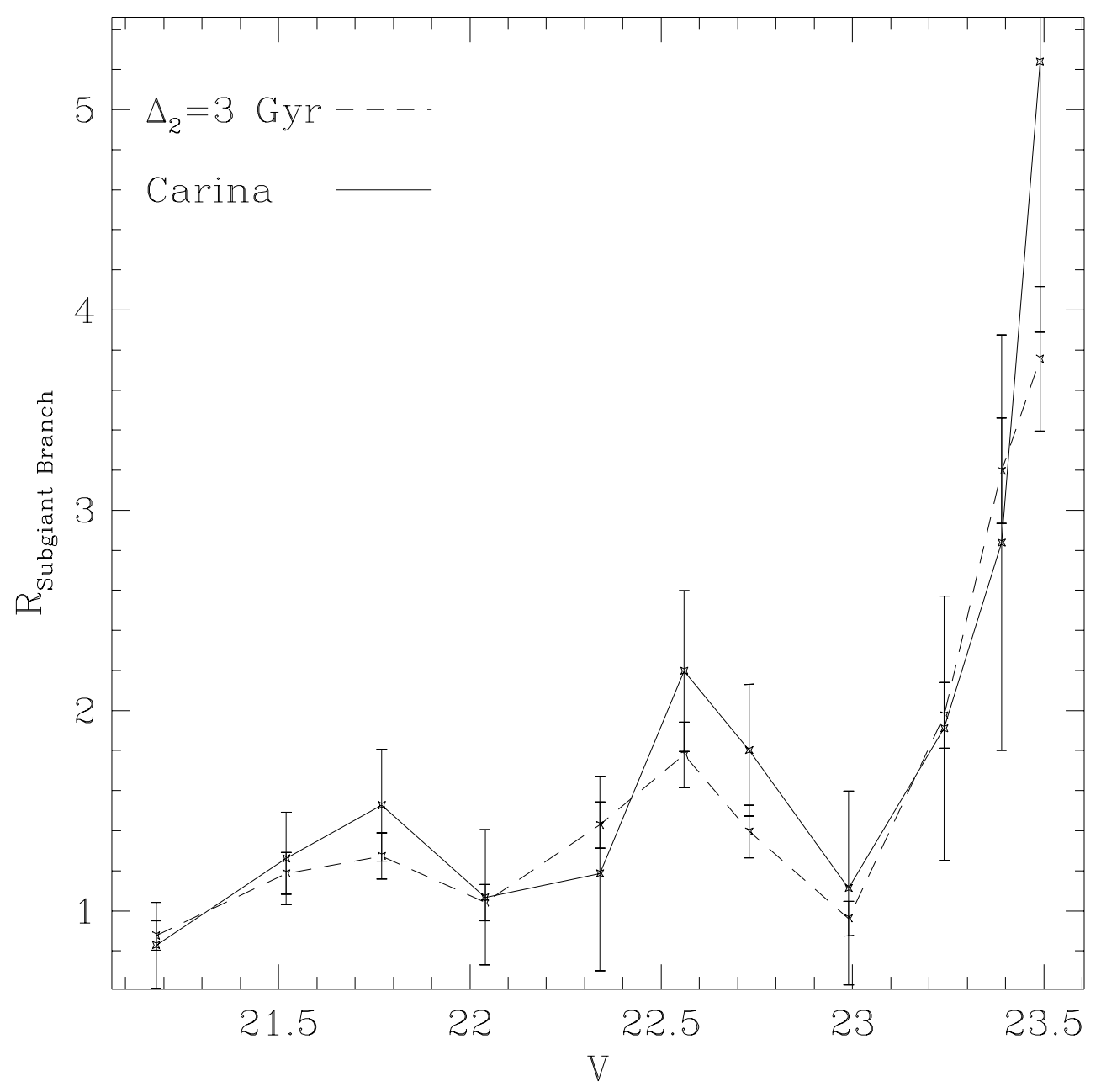

Hurley-Keller et al. - Figure 20 
Table 1. Summary of Observations

\begin{tabular}{cccccccc}
\hline \hline Field & $\alpha_{1950}$ & $\delta_{1950}$ & $N_{V}{ }^{\mathrm{a}}$ & $N_{B}^{\mathrm{a}}$ & $\begin{array}{c}t_{t o t, V}{ }^{\mathrm{b}} \\
(\mathrm{sec})\end{array}$ & $\begin{array}{c}t_{t o t, B}{ }^{\mathrm{b}} \\
(\mathrm{sec})\end{array}$ & $N_{C M D}{ }^{\mathrm{c}}$ \\
\hline 1 & $06^{h} 39^{m} 53^{s}$ & $-50^{\circ} 56^{\prime} 59^{\prime \prime}$ & 26 & 14 & 15721 & 6920 & 3051 \\
2 & $06^{h} 40^{m} 07^{s}$ & $-50^{\circ} 52^{\prime} 52^{\prime \prime}$ & 14 & 6 & 7000 & 3000 & 2233 \\
3 & $06^{h} 40^{m} 42^{s}$ & $-50^{\circ} 52^{\prime} 18^{\prime \prime}$ & 15 & 6 & 7800 & 3500 & 2025 \\
Control (1 $\left.1^{\circ} \mathrm{S}\right)$ & $06^{h} 40^{\prime}$ & $-52^{\circ}$ & 4 & 4 & 2000 & 2000 & 440 \\
\hline
\end{tabular}

${ }^{\text {a }} N_{i}$ refers to the total number of frames obtained in filter $i$

${ }^{\mathrm{b}} t_{t o t, i}$ refers to the total exposure time in filter $i$

${ }^{\mathrm{c}} N_{C M D}$ refers to the total number of stars detected in the given field 
Table 2. Sample Photometry for Field 1

\begin{tabular}{rrrcccc}
\hline \hline $\mathrm{Id}$ & $\mathrm{X}$ & $\mathrm{Y}$ & $\mathrm{V}$ & $\sigma_{V}$ & $\mathrm{~B}-\mathrm{V}$ & $\sigma_{B-V}$ \\
\hline 1 & 311.44 & 1.80 & 25.494 & 0.213 & -0.143 & 0.298 \\
8 & 66.11 & 2.23 & 24.992 & 0.125 & 0.221 & 0.250 \\
9 & 237.59 & 2.25 & 23.334 & 0.037 & 0.177 & 0.053 \\
11 & 139.60 & 2.31 & 25.443 & 0.145 & -0.043 & 0.245 \\
10 & 196.25 & 2.31 & 23.715 & 0.037 & 0.238 & 0.057 \\
12 & 85.34 & 2.41 & 25.289 & 0.172 & -0.026 & 0.243 \\
13 & 385.22 & 2.57 & 25.109 & 0.122 & 0.364 & 0.269 \\
14 & 68.34 & 2.61 & 24.540 & 0.086 & 0.283 & 0.153 \\
16 & 113.73 & 2.93 & 22.742 & 0.024 & 0.162 & 0.035 \\
18 & 63.79 & 3.05 & 23.028 & 0.035 & 0.424 & 0.056 \\
17 & 78.24 & 3.05 & 25.124 & 0.136 & 0.428 & 0.205 \\
20 & 215.23 & 3.10 & 24.046 & 0.051 & 0.274 & 0.083 \\
21 & 118.54 & 3.15 & 24.846 & 0.122 & 0.090 & 0.165 \\
23 & 277.50 & 3.27 & 24.289 & 0.076 & 0.219 & 0.114 \\
24 & 392.81 & 3.36 & 23.433 & 0.040 & 0.416 & 0.075 \\
26 & 91.67 & 3.42 & 23.632 & 0.043 & 0.493 & 0.082 \\
29 & 175.29 & 3.46 & 24.159 & 0.062 & 0.355 & 0.108 \\
31 & 387.92 & 3.60 & 25.842 & 0.253 & -0.164 & 0.402 \\
36 & 381.73 & 3.87 & 25.108 & 0.114 & 0.445 & 0.256 \\
39 & 283.09 & 3.97 & 24.173 & 0.065 & 0.203 & 0.102 \\
38 & 318.18 & 3.97 & 25.188 & 0.129 & 0.330 & 0.261 \\
40 & 126.18 & 4.06 & 24.097 & 0.066 & 0.686 & 0.136 \\
43 & 218.48 & 4.10 & 25.117 & 0.118 & 0.107 & 0.189 \\
44 & 111.47 & 4.13 & 25.301 & 0.186 & 0.072 & 0.309 \\
45 & 86.80 & 4.25 & 24.993 & 0.116 & 0.058 & 0.177 \\
46 & 58.36 & 4.42 & 24.009 & 0.053 & 0.441 & 0.124 \\
49 & 380.40 & 4.55 & 25.597 & 0.171 & -0.069 & 0.286 \\
52 & 320.41 & 4.92 & 24.480 & 0.072 & 0.655 & 0.153 \\
56 & 163.84 & 5.41 & 24.933 & 0.128 & -0.001 & 0.170 \\
57 & 309.76 & 5.49 & 24.102 & 0.049 & 0.272 & 0.090 \\
58 & 257.79 & 5.53 & 22.833 & 0.028 & 0.216 & 0.040 \\
59 & 191.60 & 5.57 & 24.933 & 0.091 & 0.565 & 0.213 \\
60 & 263.86 & 5.61 & 24.726 & 0.082 & 0.272 & 0.145 \\
\hline & & & & & &
\end{tabular}


Table 3. Magnitude and Color Errors as a Function of Magnitude.

\begin{tabular}{ccc}
\hline \hline$V$ & $\sigma_{v}$ & $\sigma_{B-V}$ \\
\hline 17.80 & 0.02 & 0.03 \\
18.80 & 0.01 & 0.02 \\
19.80 & 0.01 & 0.02 \\
20.80 & 0.01 & 0.02 \\
21.80 & 0.02 & 0.04 \\
22.80 & 0.04 & 0.08 \\
23.80 & 0.10 & 0.18 \\
24.80 & 0.16 & 0.20 \\
25.80 & 0.23 & 0.25 \\
\hline
\end{tabular}


Table 4. Blue Straggler, Horizontal Branch, and Main-Sequence Turn-Off Stars in the Carina Fields

\begin{tabular}{lcccc}
\hline \hline & BS $^{\mathrm{a}}$ & $\mathrm{BHB}^{\mathrm{b}}$ & Red Clump & $\mathrm{MSTO}^{\mathrm{c}}$ \\
\hline Field 1 & 19 & 2 & 31 & 87 \\
Field 2 & 9 & 6 & 38 & 121 \\
Field 3 & 15 & 2 & 23 & 133 \\
\hline
\end{tabular}

${ }^{a} \mathrm{BS}$ refers to blue straggler candidates.

${ }^{\mathrm{b}}$ BHB refers to blue horizontal branch stars.

${ }^{\mathrm{c}} \mathrm{MSTO}$ refers to the upper main-sequence turn-off stars. 
Table 5. Summary of Models: Ages and Durations of Episodes

\begin{tabular}{cccccc}
\hline \hline$t_{1}$ & $t_{2}$ & $t_{3}$ & $\Delta_{1}$ & $\Delta_{2}$ & $\Delta_{3}$ \\
\hline 15 & 8 & 3 & 0.5 & 2 & 1 \\
15 & 8 & 3 & 0.5 & 2 & 0.5 \\
15 & 8 & 3 & 0.5 & 1 & 1 \\
15 & 8 & 3 & 0.5 & 1 & 0.5 \\
15 & 8 & 3 & 0.5 & 0.5 & 1 \\
15 & 8 & 3 & 0.5 & 0.5 & 0.5 \\
\hline 15 & 7 & 3 & 0.5 & 2 & 1 \\
15 & 7 & 3 & 0.5 & 2 & 0.5 \\
15 & 7 & 3 & 0.5 & 1 & 1 \\
15 & 7 & 3 & 0.5 & 1 & 0.5 \\
15 & 7 & 3 & 0.5 & 0.5 & 1 \\
15 & 7 & 3 & 0.5 & 0.5 & 0.5 \\
\hline 15 & 6.5 & 3 & 0.5 & 2 & 1 \\
15 & 6.5 & 3 & 0.5 & 2 & 0.5 \\
15 & 6.5 & 3 & 0.5 & 1 & 1 \\
15 & 6.5 & 3 & 0.5 & 1 & 0.5 \\
15 & 6.5 & 3 & 0.5 & 0.5 & 1 \\
15 & 6.5 & 3 & 0.5 & 0.5 & 0.5 \\
\hline
\end{tabular}

Note. - All values are in Gyr. 
Table 6. Summary of Models: Strengths

\begin{tabular}{ccc}
\hline \hline$S_{1}$ & $S_{2}$ & $S_{3}$ \\
\hline 0.1 & 0.7 & 0.2 \\
0.1 & 0.6 & 0.3 \\
0.1 & 0.5 & 0.4 \\
\hline 0.2 & 0.7 & 0.1 \\
0.2 & 0.6 & 0.2 \\
0.2 & 0.5 & 0.3 \\
0.2 & 0.4 & 0.4 \\
\hline 0.3 & 0.6 & 0.1 \\
0.3 & 0.5 & 0.2 \\
0.3 & 0.4 & 0.3 \\
\hline
\end{tabular}

Note. - $S_{i}$ is the fraction of the total population. 
Table 7. Best Models

\begin{tabular}{|c|c|c|c|}
\hline Model & $\chi_{M S}^{2} \%^{\mathrm{a}}$ & $\chi_{S G B}^{2} \%^{\mathrm{a}}$ & $\chi^{2 \% a, b}$ \\
\hline $\begin{array}{l}t_{2}=7.0 ; \Delta_{2}=2.0 ; \Delta_{1}=1.0 \\
\quad S_{1}, S_{2}, S_{3}=.3, .5, .2\end{array}$ & 82 & 91 & 86.5 \\
\hline $\begin{array}{l}t_{2}=7.0 ; \Delta_{2}=2.0 ; \Delta_{1}=0.5 \\
\quad S_{1}, S_{2}, S_{3}=.3, .5, .2\end{array}$ & 84 & 88 & 86 \\
\hline $\begin{array}{l}t_{2}=7.0 ; \Delta_{2}=1.0 ; \Delta_{1}=1.0 \\
\quad S_{1}, S_{2}, S_{3}=.3, .5, .2\end{array}$ & 84 & 87 & 85.5 \\
\hline $\begin{array}{l}t_{2}=7.0 ; \Delta_{2}=1.0 ; \Delta_{1}=0.5 \\
\quad S_{1}, S_{2}, S_{3}=.3, .5, .2\end{array}$ & 80 & 80 & 80 \\
\hline $\begin{array}{l}t_{2}=7.0 ; \Delta_{2}=2.0 ; \Delta_{1}=1.0 \\
\quad S_{1}, S_{2}, S_{3}=.2, .6, .2\end{array}$ & 75 & 87 & 81 \\
\hline $\begin{array}{l}t_{2}=7.0 ; \Delta_{2}=1.0 ; \Delta_{1}=0.5 \\
\quad S_{1}, S_{2}, S_{3}=.2, .6, .2\end{array}$ & 72 & 90 & 81 \\
\hline $\begin{array}{l}t_{2}=7.0 ; \Delta_{2}=0.5 ; \Delta_{1}=1.0 \\
\quad S_{1}, S_{2}, S_{3}=.2, .6, .2\end{array}$ & 60 & 79 & \\
\hline $\begin{array}{l}t_{2}=6.5 ; \Delta_{2}=1.0 ; \Delta_{1}=0.5 \\
\quad S_{1}, S_{2}, S_{3}=.2, .5, .3\end{array}$ & 58 & 66 & \\
\hline $\begin{array}{l}t_{2}=7.0 ; \Delta_{2}=2.0 ; \Delta_{1}=1.0 \\
\quad S_{1}, S_{2}, S_{3}=.2, .7, .1\end{array}$ & 57 & 84 & \\
\hline $\begin{array}{l}t_{2}=6.5 ; \Delta_{2}=2.0 ; \Delta_{1}=0.5 \\
\quad S_{1}, S_{2}, S_{3}=.2, .5, .3\end{array}$ & 54 & 61 & \\
\hline $\begin{array}{l}t_{2}=7.0 ; \Delta_{2}=0.5 ; \Delta_{1}=1.0 \\
\quad S_{1}, S_{2}, S_{3}=.3, .5, .2\end{array}$ & 54 & 67 & \\
\hline $\begin{array}{c}t_{2}=7.0 ; \Delta_{2}=0.5 ; \Delta_{1}=0.5 \\
S_{1}, S_{2}, S_{3}=.3, .5, .2\end{array}$ & 48 & 80 & \\
\hline
\end{tabular}

${ }^{\mathrm{a}} \chi_{M S}^{2} \%, \chi_{S G B}^{2} \%$, and average $\chi^{2 \%}$ are actually the $\chi^{2}$ probabilities for the models computed $\chi^{2}$ value.

bThe average $\chi^{2 \%}$ is shown only for those models which passed the selection criteria. 
Table 8. Summary of Best Model Properties

\begin{tabular}{lccc}
\hline \hline SFH Parameters & $t_{2}=6.5$ & $t_{2}=7$ & $t_{2}=8$ \\
\hline$\Delta_{2}=0.5$ & 0 & 3 & 0 \\
$\Delta_{2}=1.0$ & 1 & 3 & 0 \\
$\Delta_{2}=2.0$ & 1 & 4 & 0 \\
\hline$S_{3}=0.1$ & 0 & 1 & 0 \\
$S_{3}=0.2$ & 0 & 9 & 0 \\
$S_{3}=0.3$ & 2 & 0 & 0 \\
\hline$S_{2}=S_{1}$ & 0 & 0 & 0 \\
$S_{2}>S_{1}$ & 2 & 10 & 0 \\
\hline$\Delta_{1}=0.5$ & 2 & 4 & 0 \\
$\Delta_{1}=1.0$ & 0 & 6 & 0 \\
\hline
\end{tabular}

